\title{
Correlation between the secondary structure of pre-mRNA introns and the efficiency of splicing in Saccharomyces cerevisiae Sanja Rogic*1,2, Ben Montpetit ${ }^{3,4}$, Holger H Hoos ${ }^{1}$, Alan K Mackworth ${ }^{1}$, BF Francis Ouellette ${ }^{5}$ and Philip Hieter ${ }^{3}$
}

Address: ${ }^{1}$ Department of Computer Science, University of British Columbia, Vancouver, Canada, ${ }^{2}$ Center for High-Throughput Biology, University of British Columbia, Vancouver, Canada, ${ }^{3}$ Michael Smith Laboratories, University of British Columbia, Vancouver, Canada, ${ }^{4}$ Centre for Molecular Medicine and Therapeutics, Vancouver, Canada and ${ }^{5}$ Ontario Institute for Cancer Research, Toronto, Canada

Email: Sanja Rogic* - rogic@bioinformatics.ubc.ca; Ben Montpetit - bmontpet@cmmt.ubc.ca; Holger H Hoos - hoos@cs.ubc.ca; Alan K Mackworth - mack@cs.ubc.ca; BF Francis Ouellette - francis@oicr.on.ca; Philip Hieter - hieter@msl.ubc.ca

* Corresponding author

Published: 29 July 2008

BMC Genomics 2008, 9:355

doi: $10.1|86 /| 47|-2| 64-9-355$
Received: 7 March 2008

Accepted: 29 July 2008

This article is available from: http://www.biomedcentral.com/I47I-2/64/9/355

(c) 2008 Rogic et al; licensee BioMed Central Ltd.

This is an Open Access article distributed under the terms of the Creative Commons Attribution License (http://creativecommons.org/licenses/by/2.0), which permits unrestricted use, distribution, and reproduction in any medium, provided the original work is properly cited.

\begin{abstract}
Background: Secondary structure interactions within introns have been shown to be essential for efficient splicing of several yeast genes. The nature of these base-pairing interactions and their effect on splicing efficiency were most extensively studied in ribosomal protein gene RPS I 7B (previously known as RP5 IB). It was determined that complementary pairing between two sequence segments located downstream of the 5 ' splice site and upstream of the branchpoint sequence promotes efficient splicing of the RPSI $7 B$ pre-mRNA, presumably by shortening the branchpoint distance. However, no attempts were made to compute a shortened, 'structural' branchpoint distance and thus the functional relationship between this distance and the splicing efficiency remains unknown.

Results: In this paper we use computational RNA secondary structure prediction to analyze the secondary structure of the RPSI $7 B$ intron. We show that it is necessary to consider suboptimal structure predictions and to compute the structural branchpoint distances in order to explain previously published splicing efficiency results. Our study reveals that there is a tight correlation between this distance and splicing efficiency levels of intron mutants described in the literature. We experimentally test this correlation on additional RPSI $7 B$ mutants and intron mutants within two other yeast genes.

Conclusion: The proposed model of secondary structure requirements for efficient splicing is the first attempt to specify the functional relationship between pre-mRNA secondary structure and splicing. Our findings provide further insights into the role of pre-mRNA secondary structure in gene splicing in yeast and also offer basis for improvement of computational methods for splice site identification and gene-finding.
\end{abstract}

\section{Background}

Splicing of precursor mRNA is one of the essential cellular processes in eukaryotic organisms. Although this process has been extensively studied since the discovery of splic- ing three decades ago $[1,2]$, resulting in a thorough understanding of the splicing pathway and identification of the numerous components of the splicing machinery, there are still many unanswered questions. For example, while 
the ability of pre-mRNA to form intramolecular interactions between short complementary segments in long yeast introns was initially suggested 20 years ago [3], the role of pre-mRNA secondary structure in splicing is not well understood.

Introns in S. cerevisiae are known to have bimodal length distribution [4] and can be classified into short and long introns based on their length. The distance between the 5' splice site and the branchpoint sequence, also known as the 'lariat length' or 'branchpoint distance' (we also refer to it as linear branchpoint distance), is tightly correlated with intron length (with a Pearson correlation coefficient of $r=0.99$ [5]) and can also be used to classify introns into long (5'L) and short (5'S) [3]. It was hypothesized that 5' $\mathrm{L}$ introns, for which the branchpoint distance is greater than $200 \mathrm{nt}$, can fold into secondary structures to optimize the positioning of the 5 ' splice site and branchpoint sequence to one that is optimal for spliceosome assembly [3]. This hypothesis was confirmed for a limited number of yeast introns by comprehensive biological experiments that demonstrated that the existence of such secondary structure elements is essential for splicing efficiency [6-11]. Structural elements that exhibit a similar effect on splicing efficiency were also found in introns of Drosophila melanogaster and related species [12]. Furthermore, in mammalian cells, folding of long intron sequences is facilitated by protein binding and interactions, which presumably shortens the long distance between essential splicing sequences [13].

The nature of the base-pairing interactions within introns and their effect on splicing efficiency were most extensively studied in $S$. cerevisiae's ribosomal protein gene $R P S 17 B$, previously known as $R P 51 B$ (YDR447C). It was shown that secondary structure interaction between two sequence segments located downstream of the 5 ' splice site and upstream of the branchpoint sequence promotes efficient splicing of the RPS17B pre-mRNA [7]. This interaction was further tested by comprehensive mutational and structure-probing analysis to determine the structure of the stem formed in the wildtype intron and the sensitivity of splicing efficiency to the alterations in this stem $[8,9]$. These studies demonstrated that complementary pairing between two ends of the RPS17B intron, but not necessarily the formation of the described stem, is essential for its efficient splicing in vitro and in vivo.

While the authors of the previous studies speculated that the function of the complementary pairing is to shorten the branchpoint distance, they did not attempt to determine the secondary structure of the intron and the resulting 'structural' branchpoint distance. Thus a functional relationship between this distance and the splicing efficiency remains unknown.
In this paper we use computational RNA secondary structure prediction to investigate the secondary structures of wildtype and mutant intron sequences within the $S$. cerevisiae RPS17B pre-mRNA. We present a unique algorithm for measuring 'structural' distance between two bases in an RNA secondary structure and use it to compute the distance between the 5' splice site and the branchpoint sequence based on the predicted secondary structure. Our analysis show that there is a tight correlation between structural branchpoint distances and splicing efficiency levels for all mutants examined.

\section{Results}

Secondary structure of RPS I7B intron and the efficiency of splicing

The first goal of our study was to determine if the splicing efficiency results previously reported for RPS17B intron [8] can be correlated with the computationally predicted secondary structures of wildtype and mutant intron sequences.

In this study the sensitivity of splicing to alterations in the stem formed in the RPS17B intron was tested by introducing mutations in the interacting regions designated UB1 (upstream box 1) and DB1 (downstream box 1). The assumption behind the mutant design was that any mutation within the stem would disrupt it and change the intron secondary structure in such a way that the resulting structural branchpoint distance $\left(d_{s}\right)$ would be greater than for the wildtype intron. The authors created 9 mutant introns within the RPS17B gene: $3 m U B 1$ ( 3 nt mutation), $4 m U B 1$ (4 nt), 5mUB1 (5 nt), 6mUB1 (6 nt) and 8mUB1 $(8 \mathrm{nt})$, where mutations fall in the UB1 region; $3 m D B 1$ (3 $\mathrm{nt})$ and $5 \mathrm{mDB} 1(5 \mathrm{nt})$, where mutations fall in the DB1 region and are designed to restore the base-pairing disrupted by the mutations in the $3 m U B 1$ and $5 m U B 1$, respectively; and 3mUB1_3mDB1 and 5mUB1_5mDB1, which are double mutants. All of the single mutants are expected to disrupt the secondary structure, while the double mutants are predicted to restore it. The RPS17B intron was inserted into the coding region of the copper resistance gene (CUP1), which served as a reporter gene. Thus, yeast cells grown on copper containing medium will be viable only if the intron-containing Cup1 mRNA is spliced. The results of this assay suggested that for all single mutants except $8 m U B 1$, splicing was reduced. Surprisingly, $8 m U B 1$ had a similar growth rate on copper media as the wildtype intron suggesting that splicing was as efficient. Out of two double mutants, 5mUB1_5mDB1 was able to partially rescue copper resistance, while $3 m U B 1$ 3mDB1 did not. The authors hypothesized that these unexpected results were the result of some secondary structure rearrangements; however, the secondary structure of the mutants $8 m U B 1$ and 3mUB1_3mDB1 was not explored. 
In order to investigate if the differences in the splicing efficiency levels are due to the differences in secondary structures, we computed the minimum free energy (MFE) structures of the introns using mfold $[14,15]$, one of the most frequently used RNA secondary structure prediction tools. The comparative RNA secondary structure prediction, which is considered more reliable, requires a certain number of orthologous sequences which were available only for the wildtype RPS $17 B$ intron and not for the mutants created in [8].

According to the mfold MFE predictions, the introduced mutations have the desired effect of disrupting the stem in all single mutants, but the compensatory mutations fail to restore it in two double mutants. Focusing on the positioning between the donor site and the branchpoint sequence, we compared the part of the structure that contains these two sites across all the mutants. The specified structural domain was almost identical for the $3 m U B 1$, 5mUB1，8mUB1，3mDB1，3mUB1_3mDB1 and $5 m U B 1 \_5 m D B 1$ mutants, some of which have very different splicing efficiency levels (see Additional file 1). Moreover, the full secondary structures of the $3 m U B 1$ and 3mUB1_3mDB1 mutants were almost identical with only three base-pairs difference, while the copper resistance experiment suggested significant differences in splicing efficiency. Therefore, it appears that differences in the splicing efficiency of Libri et al.'s [8] mutants cannot be attributed to differences in the computed MFE secondary structures of introns.

However, considering only a single, minimum free energy secondary structure prediction of an intron might not be the appropriate approach. While functional, non-coding RNAs, such as tRNAs and rRNAs, have a strong evolutionary pressure to maintain their unique, functional structure, it is believed that mRNAs, whose primary role is to carry the protein coding information to the translation apparatus, do not have functional constraints on their global structure. Thus, instead of always folding into unique MFE structure, it is likely that mRNAs exist in a population of structures [16-18]. Another reason for considering suboptimal structures, especially when using computational prediction methods, is that RNA secondary structure prediction algorithms have limited accuracy and sometimes the correct structure is buried among the suboptimal predictions with free energies very close to the MFE $[15,19,20]$.

Structural branchpoint distances of suboptimal secondary structures and the efficiency of splicing

Based on these considerations, we modified our approach to include not only the optimal, i.e., MFE structure, but also near-optimal predictions whose free energies are within $5 \%$ of the optimum. There is an exponential rela- tionship between the free energy of a structure and its probability in the ensemble of all possible structures for a given sequence. The probability of a structure $S_{i}$ in the Boltzmann ensemble of all possible structures $\left(S_{1}, S_{2}, \ldots\right)$ for a given RNA sequence is given by:

$$
P\left(S_{i}\right)=\frac{e^{-\Delta G\left(S_{i}\right) / R T}}{Q}
$$

where $\Delta G\left(S_{i}\right)$ is the free energy of structure $S_{i}, Q=\Sigma_{S} e^{-}$ $\Delta G(S) / R T$ the partition function for all possible secondary structures for the given sequence, $R$ is the physical gas constant, and $T$ is the temperature. The probability of a secondary structure is also called the Boltzmann weight of that structure.

From the equation we can see that the lower the free energy of a structure the higher its probability, thus, the predictions within 5\% from the MFE also represent the most probable structures for a given sequence, with the MFE prediction being the one with the highest probability.

We used RNAsubopt algorithm [20] to sample 1000 suboptimal structures within 5\% of the MFE for each considered intron. RNAsubopt first calculates all suboptimal structures within a user defined energy range and then produces a random sample of structures, drawn with probabilities equal to their Boltzmann weights. Therefore, RNAsubopt computes a representative sample of the secondary structure space within $5 \%$ of the MFE.

Since the pair-wise structure comparison and distance estimation approach that we used for MFE structure predictions were not applicable to large number of structures we had to devise a new way to quantify the structural distance between the donor site and the branchpoint sequence. We designed an algorithm that converts an RNA secondary structure into a graph and then applies a shortest-path algorithm from graph theory to compute the shortest distance between two bases in the secondary structure. To the best of our knowledge this is the first algorithm for structural distance computation. More details are given in Materials and Methods.

For each secondary structure prediction, we computed the exact distance between the donor site and the branchpoint sequences $\left(d_{s}\right)$ using the shortest-path algorithm. The average structural branchpoint distances are given in Table 1 . We assigned descriptive splicing efficiency labels based on the gel images in Figure 2A in [8]. The distributions of computed structural branchpoint distances for each of the RPS17B mutants are given in Figure 1. 
Table I: Average structural branchpoint distances for the wildtype (wt) RPS I7B intron and Libri et al.'s [8] intron mutants.

\begin{tabular}{ccc}
\hline mutant & average $\boldsymbol{d}_{s}$ & splicing efficiency \\
\hline wt & 26.67 & efficient (I) \\
3mUBI & 27.67 & slightly reduced (2) \\
5mUBI & 28.42 & slightly reduced (2) \\
8mUBI & 27.94 & efficient (I) \\
3mDBI & 37.55 & inhibited (4) \\
5mDBI & 39.19 & inhibited(4) \\
3mUBI_3mDBI & 37.44 & inhibited (4) \\
5mUBI_5mDBI & $33.8 I$ & slightly reduced (2) \\
6mUBI & 46.31 & inhibited (4) \\
4mUBI & 32.08 & reduced (3) \\
\hline
\end{tabular}

Levels of splicing efficiency were approximated from the gel images in Figure $2 \mathrm{~A}$ in [8]. The numbers within parentheses correspond to numerical values assigned to descriptive splicing efficiency labels.

These results suggest an interesting correlation between the average structural branchpoint distance and the splicing efficiency levels: sequences that are more efficiently spliced (wildtype, 3mUB1, 5mUB1, 8mUB1, 5mUB1_5mDB1, and 4mUB1) have lower values for the average distance than those that are poorly spliced. After assigning numerical values to the descriptive splicing efficiency labels (efficient $=1$, slightly reduced $=2$, reduced $=$ 3 and inhibited $=4$ ) we obtain a Pearson correlation coefficient of 0.87 .

The histograms in Figure 1 offer further insights into the relationship between structural branchpoint distances of introns and their efficiency of splicing; introns that are spliced efficiently or with slightly reduced efficiency have large frequency of suboptimal structures with $d_{s}<10$. Mutant 5mUB1_5mDB1, which does not have this prominent peak in its distribution histogram and mutant $4 m U B 1$, which has reduced splicing efficiency, but not completely inhibited, still have higher frequency of structures with $d_{s}<20$ than the remaining, poorly spliced mutants. The correlation coefficient between splicing efficiency level and the proportion of structures with $d_{s}<20$ is 0.85 .

Finally, the cumulative distribution plot of structural branchpoint distances for all mutants, where lines are labeled according to the splicing efficiency levels (efficient - blue, slightly reduced - green, reduced - black and inhibited - red) shows a clear separation of spliced and unspliced mutants (Figure 2).

Upon closer inspection we noticed that most of the structures with $d_{s}<10$ have $d_{s}=4$. Analysis of the secondary structures of these sequences reveals that this distance corresponds to a structural conformation where the donor and branchpoint sequences have two base-pairing interactions between them (see Section 2.1.3). The observed base-pairing interactions are not necessarily inconsistent with established models of the splicing process, according to which spliceosomal snRNAs interact with the donor site and the branchpoint sequence, since the base-pairing can be easily disrupted after the splicing factors have been aligned properly.

\section{Structural branchpoint distances and the efficiency of splicing for other published RPSI7B mutants}

In order to test the generality of the observed correlation between splicing efficiency levels and structural branchpoint distances we also analyzed the RPS17B intron mutants described in [9]. These are mut-UB1i, which has an inverted UB1 sequence; mut-DB1i, which has an inverted DB1 sequence; mut-UB1iDB1i, which has both UB1 and DB1 sequences inverted to make them complementary to each other; mut-5, which reduces the consecutive pairing region to 5 base-pairs, mut-12; which improves pairing to 12 consecutive base-pairs (eliminating one one-nucleotide bulge); and mut-18, which extends pairing to 18 consecutive base-pairs (eliminating all three bulges in the pairing region). The authors compared splicing efficiency of the wildtype and mutant introns by analyzing the formation of spliceosomal complexes. Based on their gel images, we assigned descriptive and numerical splicing efficiency labels to the tested sequences (see Table 2 ). The average structural branchpoint distances of 1000 suboptimal structures sampled from within 5\% of the MFE for each mutant are given in Table 2.

The branchpoint distance results for these mutants are similar to those of Libri et al.'s [8] mutants; the average structural branchpoint distances are lower for the sequences that are efficiently spliced (wildtype, mutUB1iDB1i, mut-12, and mut-18). After assigning numerical values to the descriptive splicing efficiency labels (improved $=1$, normal $=2$ and reduced $=3$ ), we obtain the correlation coefficient as 0.85 . This, again, corresponds to the ability of these sequences to fold in such a way as to bring the donor site and the branchpoint sequences close to each other; each of the efficiently spliced sequences has a large fraction of predicted secondary structures for which $d_{s}<10$ (Figure 3 and Additional file 2). The mutants that show reduced splicing have very few of these structures $(0.02 \%$ for $m u t-U B 1 i$ and $0.33 \%$ for $m u t-5)$, except for $m u t-D B 1 i$, which has $11.3 \%$ of structures with $d_{\mathrm{s}}<10$. However, this is still significantly lower than for the efficiently spliced mutants. Again, the cumulative distribution plot clearly separates mutants based on their splicing efficiency (Figure 3).

\section{Base-pairing probabilities of the RPS I 7B intron and the efficiency of splicing}

The branchpoint distance analysis of S. cerevisiae's RPS17B intron suggests that the ability to form highly probable 


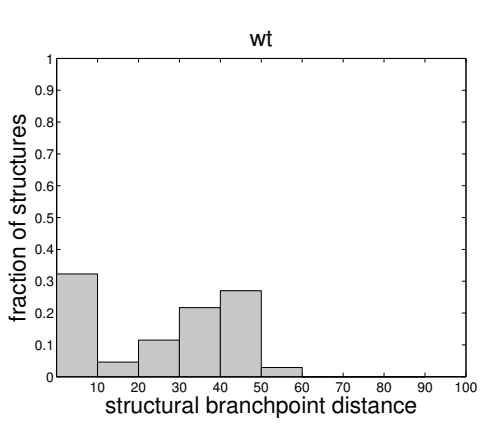

(a)

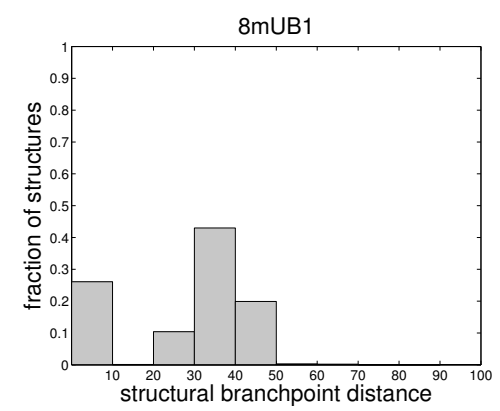

(d)

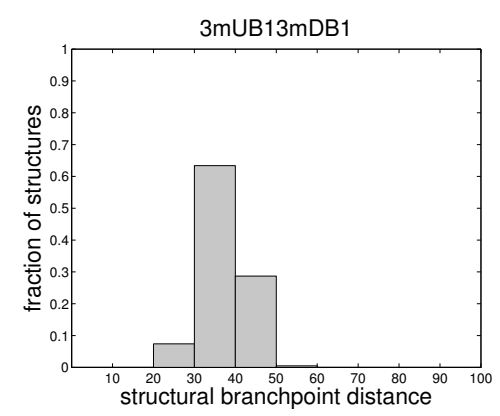

(g)

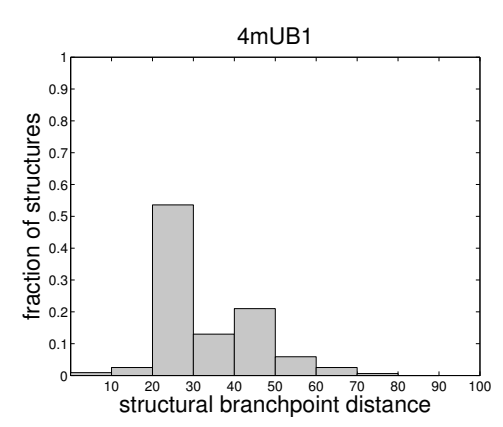

(j)

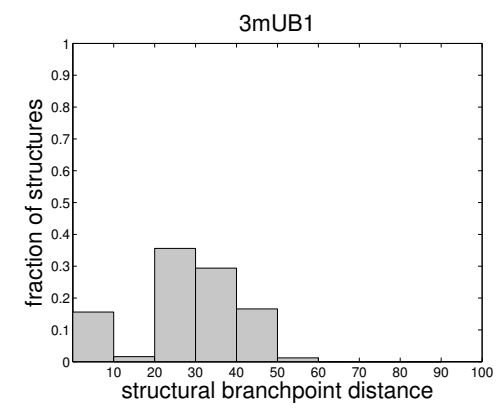

(b)

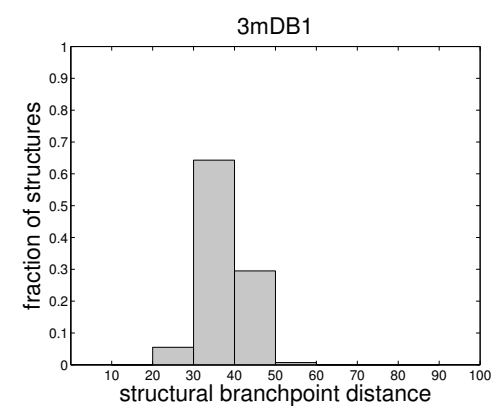

(e)

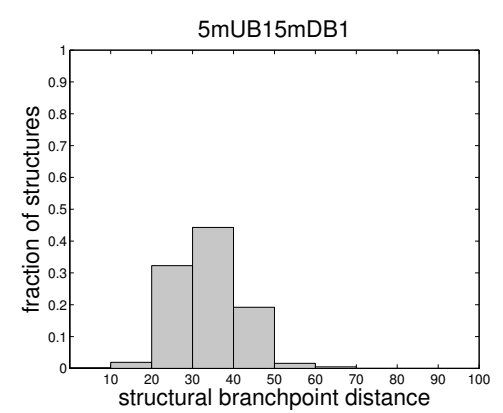

(h)

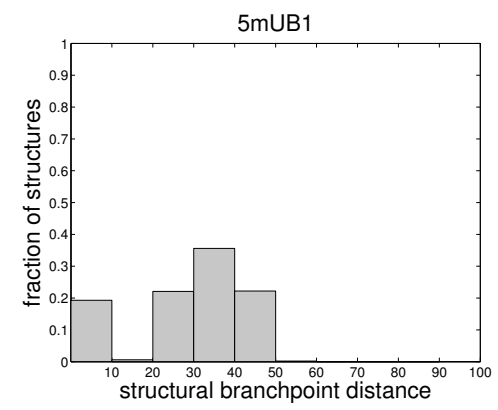

(c)

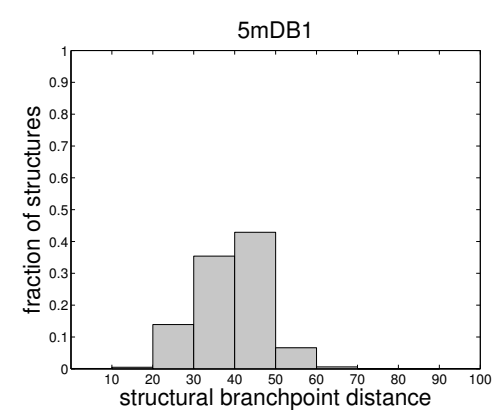

(f)

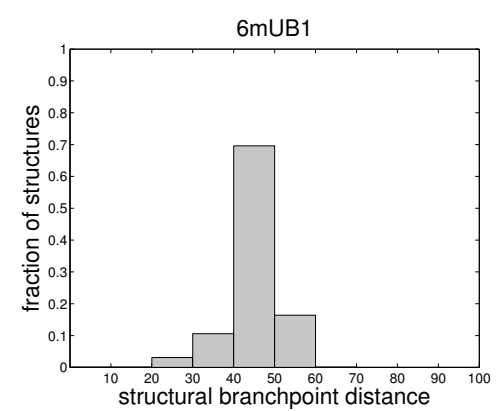

(i)

Figure I

Distribution histograms of structural branchpoint distances for (a) wt, (b) $3 m U B I$, (c) $5 m U B I$, (d) $8 m U B I$, (e) $3 m D B I$, (f) $5 m D B I$, (g) 3mUBI_3mDBI, (h) $5 \mathrm{mUBI} 5 \mathrm{mDB} I$, (i) $6 \mathrm{mUBI}$, and (j) $4 \mathrm{mUBI}$ introns. 


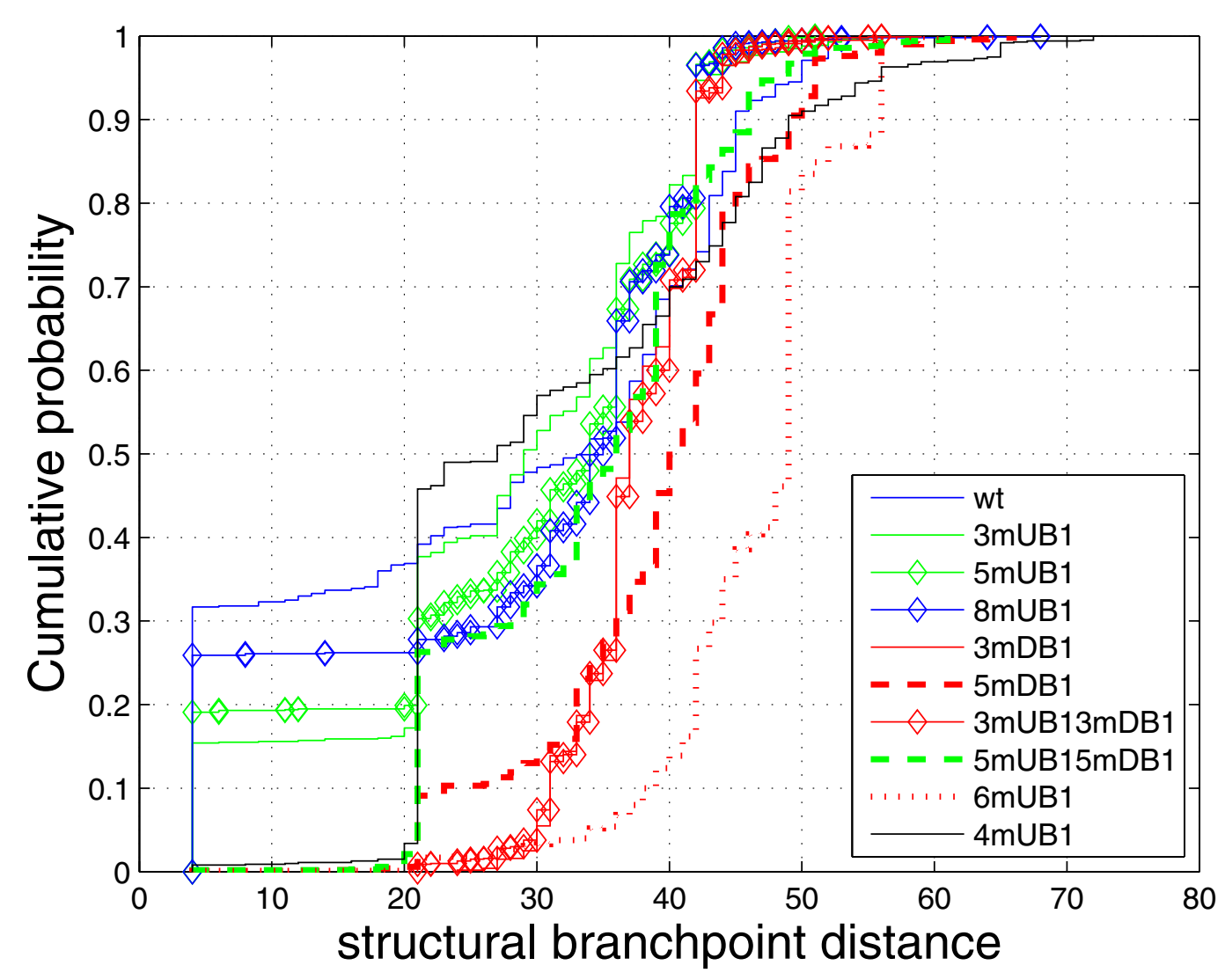

Figure 2

Cumulative distributions of structural branchpoint distances for all Libri et al.'s [8] intron mutants.

secondary structures (within 5\% of the MFE) with short distance between the donor site and the branchpoint sequence seems to be required for efficient splicing of the intron. The short structural branchpoint distance for the $R P S 17 B$ intron results from two base-pair interactions: between the first intron base $(G)$ and the third base of the

Table 2: Average structural branchpoint distances for the wildtype (wt) RPSI7B intron and Charpentier and Rosbash's [9] intron mutants.

\begin{tabular}{ccc}
\hline mutant & average $\boldsymbol{d}_{\boldsymbol{s}}$ & splicing efficiency \\
\hline wt & 26.67 & normal (2) \\
mut-UB Ii & 42.51 & reduced (3) \\
mut-DBIi & 35.95 & reduced (3) \\
mut-UBIiDB Ii & 26.39 & improved (I) \\
mut-5 & 32.14 & reduced (3) \\
mut-12 & 24.82 & improved (I) \\
mut-18 & 25.30 & improved (I)
\end{tabular}

We inferred levels of splicing efficiency based on Figures 2 and 3 and Table I in [9]. The numbers within parentheses correspond to numerical values assigned to descriptive splicing efficiency labels. branchpoint sequence $(\mathrm{C})$; and between the second base in the intron (U) and the second base of the branchpoint sequence (A) (see Figure 4). It is possible to compute the probability of these base-pairing interactions directly using a dynamic programming algorithm that computes the partition function [21]. The base-pair probability reflects a sum of all probability-weighted structures in which the chosen base-pair occurs. Thus, these base-pairing probabilities also take into account the structures that were not within 5\% from the MFE, eliminating the necessity to chose an arbitrary percent suboptimality value. The base-pair probabilities can be computed using RNAfold [22], another frequently used program for RNA secondary structure prediction.

The base-pair probability values for the wildtype RPS17B intron and all of Libri et al.'s [8] mutants are given in Table 3. The probability values for the two base-pairs (G$\mathrm{C}$ and U-A) are identical up to second decimal place for each intron sequence and that is why only one number is shown in the table. It can be observed that all of the effi- 


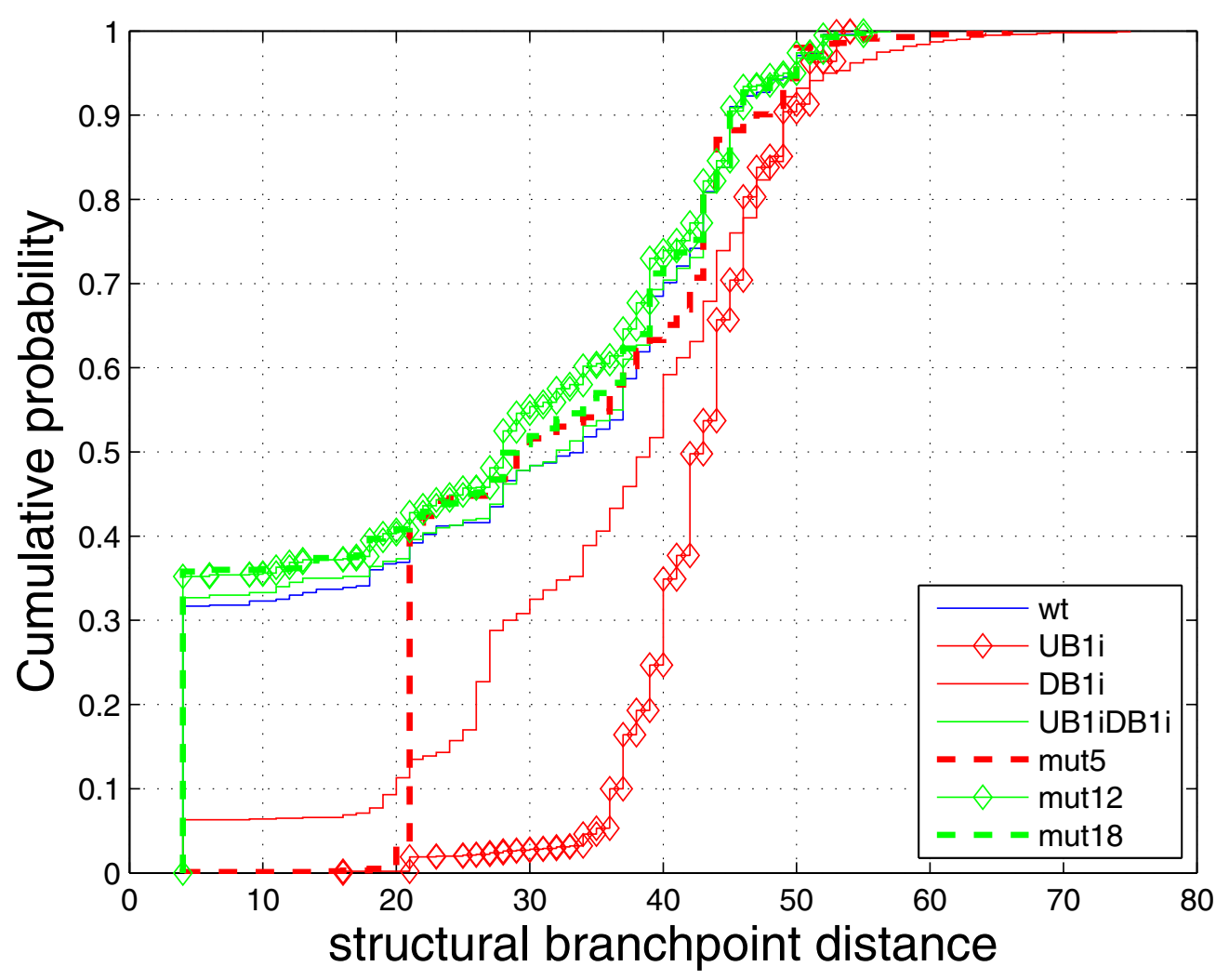

\section{Figure 3}

Cumulative distributions of structural branchpoint distances for all Charpentier and Rosbash's [9] intron mutants.

ciently spliced sequences have higher base-pair probabilities than the poorly spliced sequences $(r=-0.92)$. The correlation is not strictly linear since, for example, the mutant sequence $8 m U B 1$ has almost the same base-pair probability value as $3 m U B 1$ and $5 m U B 1$, although it is more efficiently spliced than these two. Similarly, the double mutant $5 m U B 1 \_5 m D B 1$ is more efficiently spliced than $4 m U B 1$, but this is not reflected in the base-pair probability values.

For Charpentier and Rosbash's mutants, the base-pair probabilities are also higher for the sequences that are more efficiently spliced (Table 4): all of the sequences that are efficiently spliced (wildtype, mut-UB1iDB1i, mut-12, and $m u t-18$ ) have base-pair probabilities of 0.40 , while the other sequences have lower values $(r=-0.85)$.

Overall, based on the results for Libri et al.'s [8] and Charpentier and Rosbash's [9] mutants it seems that, at least for RPS17B intron, base-pair probabilities for the two base-pairs formed between the first two bases of the intron and the second and third base of the branchpoint sequence are good indicators of splicing efficiency. We will see in the following sections that this is not a general requirement for all genes. Taken together with the observed correlation between the splicing efficiency levels and structural branchpoint distances the results are consistent with the following hypothesis: the existence of highly probable secondary structures that have short branchpoint distance is required for efficient splicing of yeast

introns.

\section{Experimental testing of the hypothesis}

In order to test the validity of the proposed hypothesis, we designed and functionally tested in vivo a series of RPS17B intron mutants. To assay the effect of these mutations on splicing we opted to introduce the mutated intron sequences at their endogenous locus, instead of within the CUP1 gene as was previously done $[8,9]$. This allows us to analyze the splicing of this intron within its normal context of flanking DNA sequences. We estimated the splicing efficiency directly from protein expression levels, which were quantified using a fluorescence imaging system.

Using protein expression as a measurement of splicing efficiency requires that: 1) the level of protein abundance 


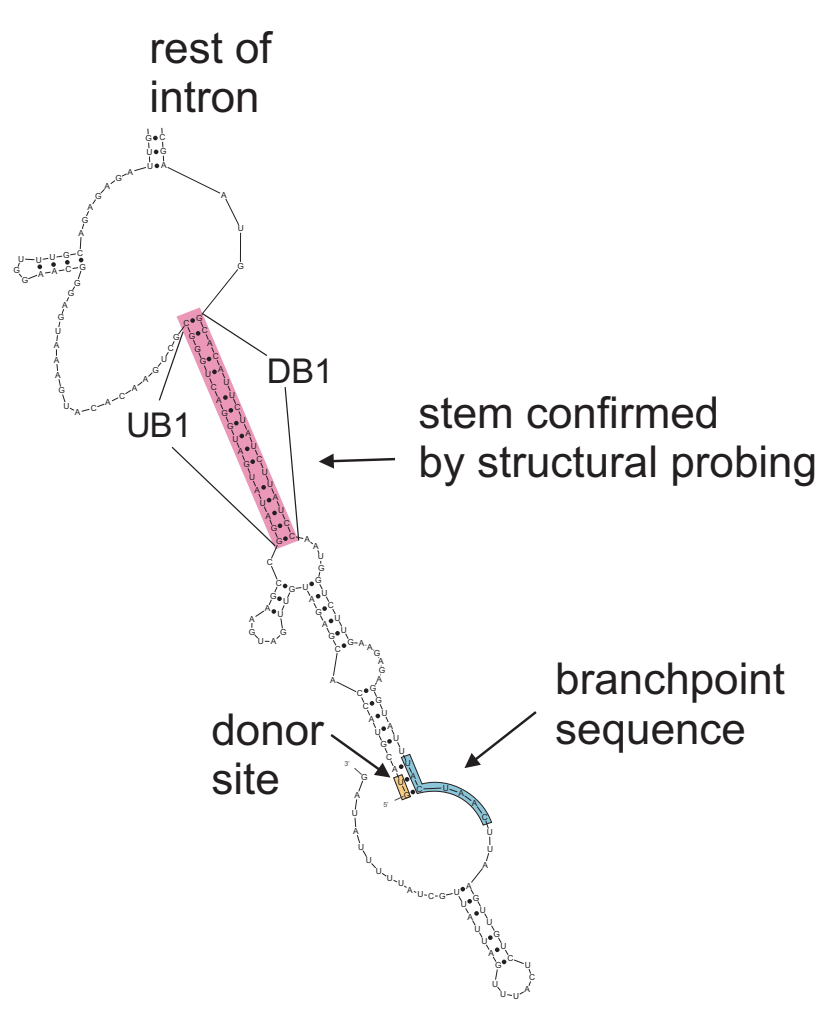

Figure 4

A part of the wildtype RPSI TB intron secondary structure that shows base-pairing between the donor site and the branchpoint sequence. The highlighted stem is the same as the one identified in [9] using experimental structure probing.

is proportional to the mRNA abundance (for a given gene) in the cell and, 2) the abundance of mRNA in the cell reflects any change in splicing efficiency. To demonstrate that $R P S 17 B$ follows these general rules, we analyzed a number of Libri et al.'s [8] mutants that have previously documented changes in mRNA levels for their protein expression levels. The sequences tested were the wildtype $R P S 17 B$ intron, and the $5 m U B 1,3 m U B 1$, $8 m U B 1,5 m D B 1$, and 3mDB1 mutated introns. The levels of protein expression, as shown in Figure 5, are proportional to the levels of copper-resistance in the copper growth assay in [8]. Moreover, our approach is able to provide a quantifiable measure for mutants such as $3 m D B 1$ and $5 m D B 1$, which did not support any growth in the copper growth assay. Thus, using changes in protein expression levels in the context of different intron sequences to assay the effects of mutations on splicing efficiency is a valid approach.

\section{New RPSI7B intron mutants}

We designed 8 new RPS17B intron mutants for the purpose of testing our current model of correlation between intronic pre-mRNA secondary structure and splicing efficiency. The most important structural characteristic used for mutant design was structural branchpoint distance $\left(d_{s}\right)$ of its MFE and suboptimal structures. Four mutants that are predicted to splice efficiently were designed to have multiple suboptimal structures with contact conformation (Figure 4) and short average structural branchpoint distance (these mutants are labeled with letter ' $S$ ', which stands for short $d_{s}$ ). The only exception is mutant $r p s 17 b$ $S 2$, which does not have any suboptimal structures with contact conformation, but still exhibits a short structural branchpoint distance (most of the suboptimal predictions have $d_{s}=10$ ). This mutant was designed to test whether contact conformation, rather than the resulting short structural branchpoint distance, is important for splicing. Four mutants that are predicted to have reduced splicing were designed not to have any structures with contact conformation or otherwise short structural branchpoint distances (these mutants are labeled with letter 'L', which stands for long $d_{s}$ ).

The mutant design was based on mfold predictions, while RNAsubopt predictions where used post-experimentally to analyze the results. Mfold also samples the suboptimal space of secondary structures, however it does not compute all possible structures and the sample is much smaller. Although the distribution of $d_{s}$ computed based on structure predictions by mfold is similar to the one based on RNAsubopt predictions, the average distances for RNAsubopt predictions are not as distinct between ' $S$ ' and 'L' mutants as ones based on mfold predictions.

Table 5 shows average $d_{\text {s }}$ for newly designed mutants based on RNAsubopt predictions and base-pair probabilities computed by RNAfold. The analogous table based on mfold suboptimal predictions, which was used in the design process is given in Additional file 3.

As seen in Figure 6, mutants $r p s 17 b-L 1, r p s 17 b-L 2$ and $r p s 17 b-L 4$ have reduced protein expression levels when compared to the wildtype as expected. Mutant $r p s 17 b-L 3$ has reduced splicing efficiency but not as much as the other three mutants with long structural branchpoint distances. As previously explained, this mutant was designed to have reduced splicing based on suboptimal predictions by mfold, which failed to predict any structures with $d_{s}<$ 10. However, RNAsubopt, which does a more rigorous sampling of the suboptimal space, detected a small fraction of suboptimal structures that have $d_{s}<10$ (see Additional file 4). This is in agreement with the relatively high probability of base-pairing interaction between the donor site and the branchpoint sequence (0.21).

Mutants $r p s 17 b-S 1, r p s 17 b-S 2$, and $r p s 17 b$-S3 are all spliced efficiently, as predicted. The efficient splicing of mutant 
Table 3: Base-pairing probabilities of contact conformation (Figure 4) for the wildtype (wt) RPSI $7 B$ intron and Libri et al.'s [8] intron mutants.

\begin{tabular}{ccc}
\hline mutant & base-pairing probability & splicing efficiency \\
\hline wt & 0.40 & efficient \\
$3 m U B I$ & 0.33 & slightly reduced \\
$5 m U B I$ & 0.31 & slightly reduced \\
$8 m U B I$ & 0.34 & efficient \\
$3 m D B I$ & 0.01 & inhibited \\
$5 m D B I$ & $<0.01$ & inhibited \\
$3 m U B I+3 m D B I$ & 0.01 & inhibited \\
$5 m U B I 5 m D B I$ & 0.11 & slightly reduced \\
6mUBI & 0.05 & inhibited \\
$4 m U B I$ & 0.18 & reduced \\
\hline
\end{tabular}

rps17b-S2, which has short structural branchpoint distance $\left(d_{s}=10\right)$ without contact conformation in many of the predicted structures, suggests that a specific structural arrangement between the donor site and the branchpoint sequence is not required for efficient splicing. Mutant $r p s 17 b$-S4 shows reduced levels of protein abundance, which is in disagreement with our prediction. The mutated sequence for this mutant has the same location as the mutated sequence for the mutant $r p s 17 b-S 3$, which is efficiently spliced, thus we can exclude the possibility that the discrepancy in splicing is sequence-based. A possible explanation for this phenomenon may be the existence of a very thermodynamically stable stem (with free energy $\Delta G=-36.6 \mathrm{kcal} / \mathrm{mol}$ ) that holds the 5 ' splice site and the branchpoint together (analogous stems in wildtype introns have much higher free energy, see Section 2.3). This stem may be too stable to be disrupted, which might prevent the spliceosome to bind to the splice signals [8]. Overall, the results on the new RPS17B intron mutants are consistent with the proposed model of the role of intronic secondary structure in gene splicing in yeast.

Selecting additional genes for experimental validation

To further validate our hypothesis regarding the role of intron secondary structure in splicing, we selected addi-

Table 4: Base-pairing probabilities of contact conformation for the wildtype (wt) RPSI7B intron and Charpentier and Rosbash's [9] intron mutants.

\begin{tabular}{ccc}
\hline mutant & base-pairing probability & splicing efficiency \\
\hline wt & 0.40 & normal \\
mut-UB Ii & 0.04 & reduced \\
mut-DBIi & 0.25 & reduced \\
mut-UB IiDB Ii & 0.40 & improved \\
mut-5 & 0.04 & reduced \\
mut- I2 & 0.40 & improved \\
mut- I8 & 0.40 & improved \\
\hline
\end{tabular}

tional yeast, intron-containing genes to test our model. The selection criteria were: the linear distance (number of nucleotides) between the donor site and the branchpoint sequence is greater than $200 \mathrm{nt}$ ( $5^{\prime} \mathrm{L}$ introns); the intron does not contain an snRNA gene; the gene is not essential (i.e., cells are viable if the gene is mutated or deleted); and the protein product has relatively high abundance in the cell, is amenable to c-terminal tagging, and has molecular weight between $20-120 \mathrm{kDa}$ (to facilitate manipulation).

From our initial dataset of 98 yeast genes that contain $5^{\prime} \mathrm{L}$ introns (see Materials and Methods), 18 genes matched the selection criteria (17 of these were ribosomal protein genes). We selected two of these for the experiments: the ribosomal protein gene RPS6B (YBR181C) and the amino-peptidase gene APE2 (YKL157W).

The RPS6B gene contains one intron of length $352 \mathrm{nt}$, with a linear branchpoint distance (the distance between the 5 ' splice site and the branchpoint sequence) of $d=329$ nt. The computed structural branchpoint distance $\left(d_{\mathrm{s}}\right)$ is 18 for the MFE and all the suboptimal computationally predicted secondary structures within $5 \%$ of the MFE. Thus for this intron, unlike for the RPS17B intron, the donor and branchpoint sequences are not base-paired.

The APE2 gene contains one intron of length $383 \mathrm{nt}$, with a linear branchpoint distance of $d=327 \mathrm{nt}$. One of the suboptimal structures within 5\% of the MFE has a structural branchpoint distance of 6 and the others have greater distances. In the suboptimal prediction that has $d_{s}=6$ there is no base-pairing interactions between the donor and branchpoint sequences.

\section{RPS6B intron mutants}

We designed intron mutants for the RPS6B gene in a similar manner as for the RPS17B gene: the mutants that are supposed to have efficient splicing were designed to have similar structural branchpoint distances as the wildtype intron, and the mutants that are supposed to have reduced splicing were designed to have longer distances (see Additional file 5). Table 6 shows average structural branchpoint distances for a sample of 1000 suboptimal predictions within 5\% of the MFE and the probability of short branchpoint distance derived form the base-pairing probabilities. The reported probability is the highest basepair probability between the first donor nucleotide and any nucleotide within 20 bases away from the branchpoint adenosine. This guarantees that the branchpoint distance in a secondary structure that contains that basepair will be no longer than 20 .

From Figure 7 we can see that all of the 'S' mutants, which have structural branchpoint distances similar to the wildtype intron, are expressed at levels similar to the 


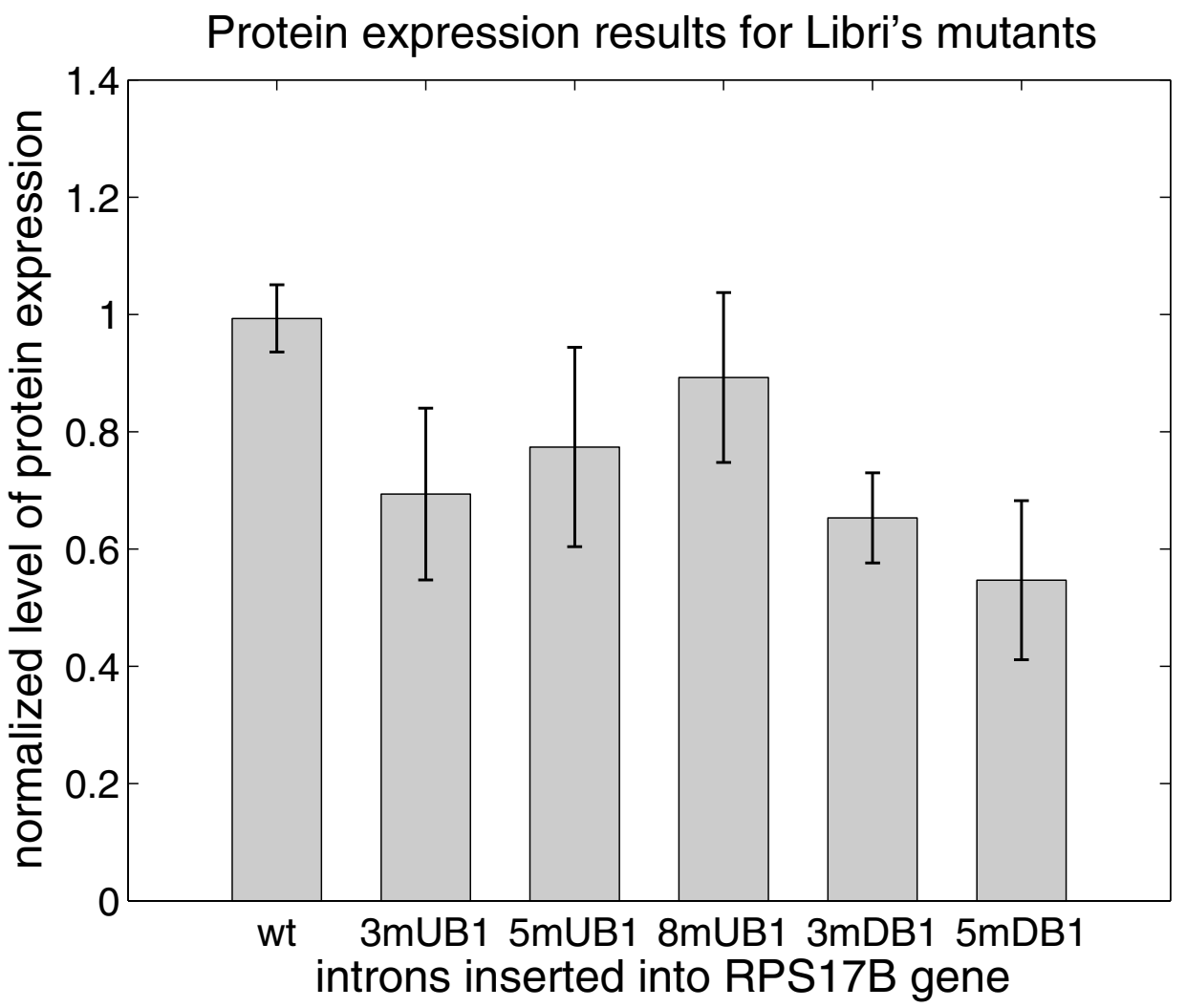

\section{Figure 5}

Protein expression levels for the RPS I 7B gene containing some of Libri et al.'s [8] mutant introns. Expression levels are normalized with respect to the internal loading control and plotted as a fraction of the wildtype expression level. Shaded boxes represent the mean value for several different samples and error bars represent +/- I standard deviation for these samples. The error bar for the wildtype intron comes from the comparison of two different wildtype samples.

wildtype. Mutant $r p s 6 b$ - L1, which has $\operatorname{avg}\left(d_{s}\right)=40$ shows a reduction in splicing efficiency. The probability of $d_{s}<$ 20 also correlates well with the protein expression data except for mutant $r p s 6 b$-S5 for which $d_{s}>20$ for all suboptimal predictions. Thus, for the RPS6B gene, structural branchpoint distances slightly longer than 20 seem to be still optimal for splicing. To summarize, the protein expression data for the RPS6B gene containing designed intron mutants are compatible with our proposed model of splicing efficiency dependence on the structural branchpoint distance.

\section{APE2 intron mutants}

Using the same selection criteria as before, we designed six APE2 intron mutants. The values for average $d_{s}$ and the probabilities of structural branchpoint distance shorter than 20 are given in Table 7, and the histograms of structural branchpoint distance distributions are given in Additional file 6 .
The experimental results are consistent with our prediction for five out of seven mutants: mutants ape2-S1, ape2S2, ape2-S3 and ape2-S5 all have a level of protein abundance similar to the wildtype (Figure 8) and mutant ape2L1 shows significantly reduced expression as expected. Mutant ape2-L2, which was expected to have reduced protein abundance as a consequence of reduced splicing efficiency, is expressed at the same level as the wildtype. Also, mutant ape2-S4 has reduced splicing despite the fact that it has a similar distribution of structural branchpoint distances as the wildtype intron. Since this mutant has the mutation at the same location as ape2-L1 (see Materials and Methods), it is possible that the intron segment that we mutated was important for splicing (e.g., contained a splicing enhancer). Overall, the results for APE2 mutants support our hypothesis of the role of structural branchpoint distance in gene splicing.

\section{Shortening of branchpoint distances by zipper stems}

The splicing efficiency study of RPS17B, RPS $6 B$ and APE2 genes containing wildtype and mutant introns supports 
Table 5: Characteristics of newly designed RPSI7B mutants.

\begin{tabular}{ccc}
\hline mutant & avg(ds) & bp prob \\
\hline wt & 26.67 & 0.40 \\
rpsI7b-LI & 43.63 & 0.0 \\
rpsI7b-L2 & 41.11 & 0.0 \\
rpsI7b-L3 & 34.05 & 0.21 \\
rpsI7b-L4 & 32.98 & 0.04 \\
rpsI7b-SI & 24.55 & 0.40 \\
rpsI7b-S2 & 29.62 & 0.03 \\
rpsI7b-S3 & 12.65 & 0.80 \\
rpsI7b-S4 & 9.27 & 0.70
\end{tabular}

$\operatorname{avg}\left(d_{s}\right)$ - average structural branchpoint distances of 1000 suboptimal structures predicted by RNAsubopt; bp prob - basepairing probability of interaction between the donor site and the branchpoint sequence based on the partition function.

our hypothesis that short structural branchpoint distances are required for efficient splicing. Although these distances are computed in the context of the secondary structure of the entire intron, our hypothesis is still consistent with the original hypothesis [3] that attributes the shortening of a long branchpoint distance to a single stem.
Such stems, which we will refer to as 'zipper' stems, since they 'zip' the intron, are probably essential for achieving a short structural branchpoint distance. If we analyze the computed secondary structures of the RPS17B, RPS $6 B$ and APE2 wildtype introns we can easily identify stable stems whose $3^{\prime}$ and $5^{\prime}$ constituents are close to the donor site and the branchpoint sequence (Figure 9). The zipper stem labeled in the RPS17B intron is the same as the one identified in [9] using experimental structure probing.

To further test the functional importance of the identified zipper stems we performed comparative structure analysis using several closely related yeast species ( $S$. paradoxus, $S$. mikatae, and S. bayanus, as well as $S$. cerevisiae, all belonging to the Saccharomyces sensu stricto group). We used multiple sequence alignments to extract the orthologous intron sequences for our three genes $[23,24]$. Both $R P S 17 B$ and RPS6B intron alignments contain three sensu stricto sequences. The multiple sequence alignment for APE2 contains all four sequences; however, these are not intronic sequences but sequences from the exon 2 of the APE2 gene. This error is due to the old S. cerevisiae anno-

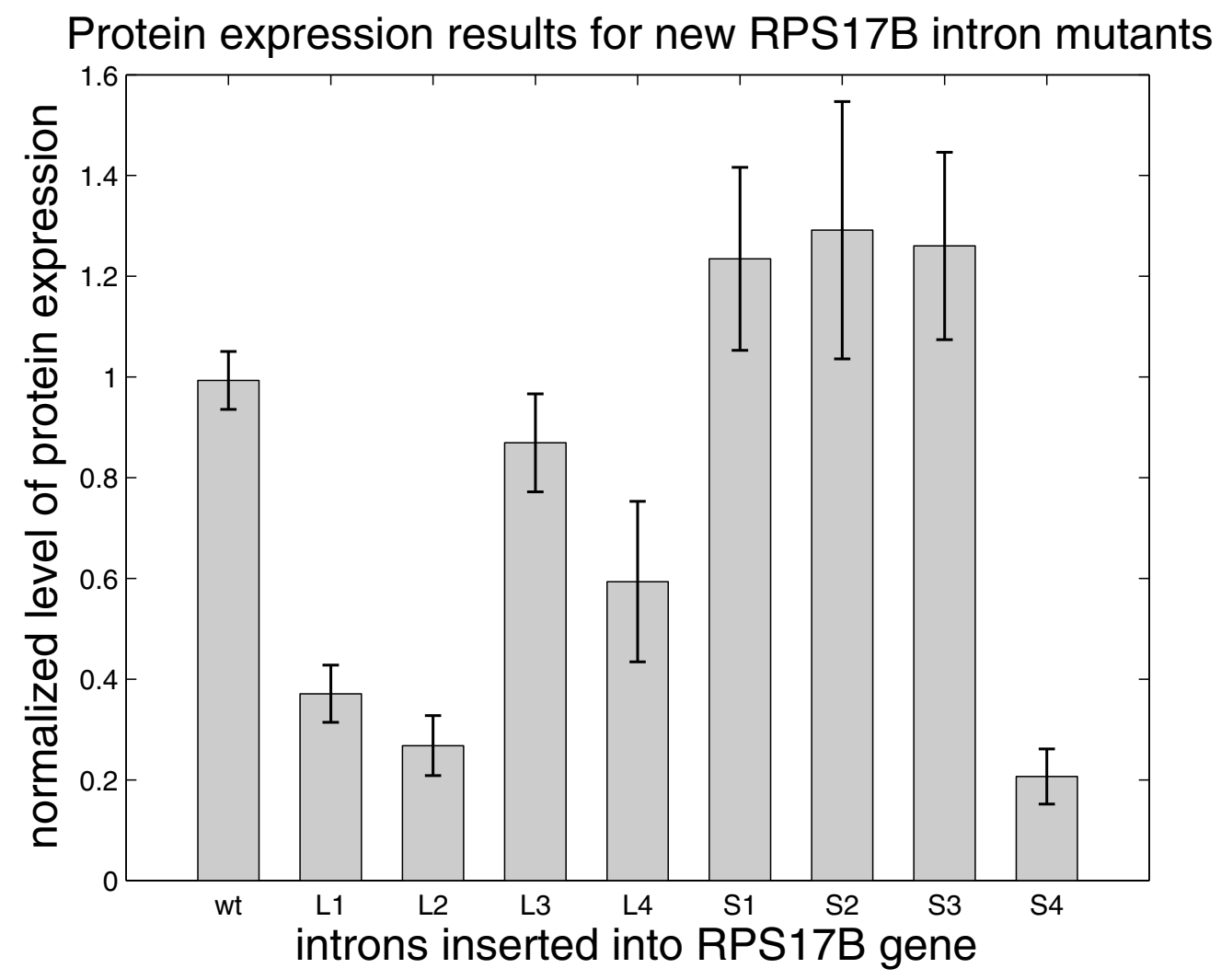

Figure 6

Protein expression results for the RPSI 7B gene containing the newly designed mutant introns. 
tation which mapped two genes to the location of the current APE2 gene [25].

We computed the consensus structure of RPS17B and RPS6B introns using Alifold [26]. The previously indicated zipper stems were found in the consensus structures for both genes (Figure 9), thus suggesting evolutionary conservation of these structural elements.

\section{Discussion}

The hypothesis that secondary structure interactions within yeast introns are needed for efficient splicing was proposed two decades ago [3]. Since then, experimental evidence in support of this hypothesis was found for several of S. cerevisiae's introns [6-11]. These studies identified complementary segments located downstream of the donor site and upstream of the branchpoint sequence whose base-pairing interactions are essential for splicing. It is conjectured that the function of the formed stem is to bring the donor site and the branchpoint sequence closer together so that they are in optimal alignment for spliceosome assembly.

In this paper we use computational RNA secondary structure prediction to study structural requirements for efficient splicing in yeast. Our approach considers a representative sample of suboptimal structures with free energies close to the MFE and it also considers the entire secondary structure of an intron, rather than a single stem, both of which are more consistent with the nature of RNA molecules. Furthermore, the approach includes a calculation of the structural branchpoint distance, which is used to quantify the effect of the secondary structure on the distance between the donor site and the branchpoint sequence and can easily be correlated with splicing efficiency measurements. Using this method we were able to identify structural characteristics of the RPS $17 B$ intron and its mutants that seem to be responsible for their splicing differences. Notably, mutants that are likely to have a short structural branchpoint distance are spliced more efficiently.

Table 6: Characteristics of newly designed RPS6B mutants.

\begin{tabular}{ccc}
\hline mutant & avg(ds) & bp prob \\
\hline wt & 18.06 & 0.84 \\
rps6b-LI & 36.74 & 0 \\
rps6b-SI & 19.08 & 0.65 \\
rps6b-S2 & 18.04 & 0.84 \\
rps6b-S3 & 18.04 & 0.83 \\
rps6b-S4 & 18.09 & 0.84 \\
rps6b-S5 & 22.00 & 0
\end{tabular}

$\operatorname{avg}\left(d_{s}\right)$ - average structural branchpoint distance; bp prob probability of $d_{s}<20$.
Based on our model of structural requirements for efficient splicing we computationally designed intron mutants for three S. cerevisiae genes, RPS17B, RPS6B and $A P E 2$, and experimentally tested their splicing efficiency. The results were mostly consistent with our model, with a few exceptions (rps17b-L3, rps17b-S4, ape2-L1 and ape2S4) which may be due to some structural characteristics of mutants that are not considered by the current model or some inherent approximations in the model that are discussed below. Some of the intron mutants that were designed to have different structural characteristics and splicing efficiencies have mutations at the same locations (e.g., $r p s 17 b$-L3 and $8 m U B 1 ; r p s 17 b$-S3 and 3mDB1; rps6b$L 1$ and $r p s 6 b-S 3)$. The experimental results that confirm differences in splicing between these pairs of mutants indicate that the secondary structure of a pre-mRNA, rather than the underlying primary sequence, is responsible for differences in splicing.

We also tested our model on the YRA1 gene intron, whose splicing efficiency had previously been studied by Preker and Guthrie [27]. The published experimental results were in agreement with our model; the efficiently spliced mutants ( $\triangle L 10$ and $\triangle T C C / G G A$ ) had higher base-pair probabilities than the poorly spliced sequences (wildtype intron and mutants $\Delta R / L 10, T C C \Delta L 10, G G A \Delta L 10$ and TCC+GGA $\triangle L 10$ ) (data not shown).

Our current model is simplified in the sense that the secondary structure of an intron is computed disregarding its flanking sequences, and the three dimensional branchpoint distance is estimated from secondary structure interactions. However, we believe that folding intronic sequences in isolation is appropriate, partly because of the existence of co-transcriptional splicing, where splicing occurs before the entire pre-mRNA has been synthesized [28-30]. Therefore, the precise part of the pre-mRNA that serves as the splicing substrate is not known. The region upstream of the transcribed intron, which consists of the $5^{\prime}$ UTR and the first exon, is also not precisely defined due to the fact that the transcription start sites have not been unambiguously mapped [31]. In addition, 5'UTRs are known to associate with a number of protein factors $[32,33]$ which are likely to have an effect on the structure formation, but these interactions are not currently modelled by computational RNA secondary structure approaches. A preliminary investigation, in which we considered some of the upstream region yielded inconclusive results (data not shown). Thus, we believe that folding only intronic sequences gives us a reasonable approximation of the secondary structure of an intron at the time of the splicing reaction.

The approximation of the three dimensional branchpoint distance using pre-mRNA secondary structure is necessary 


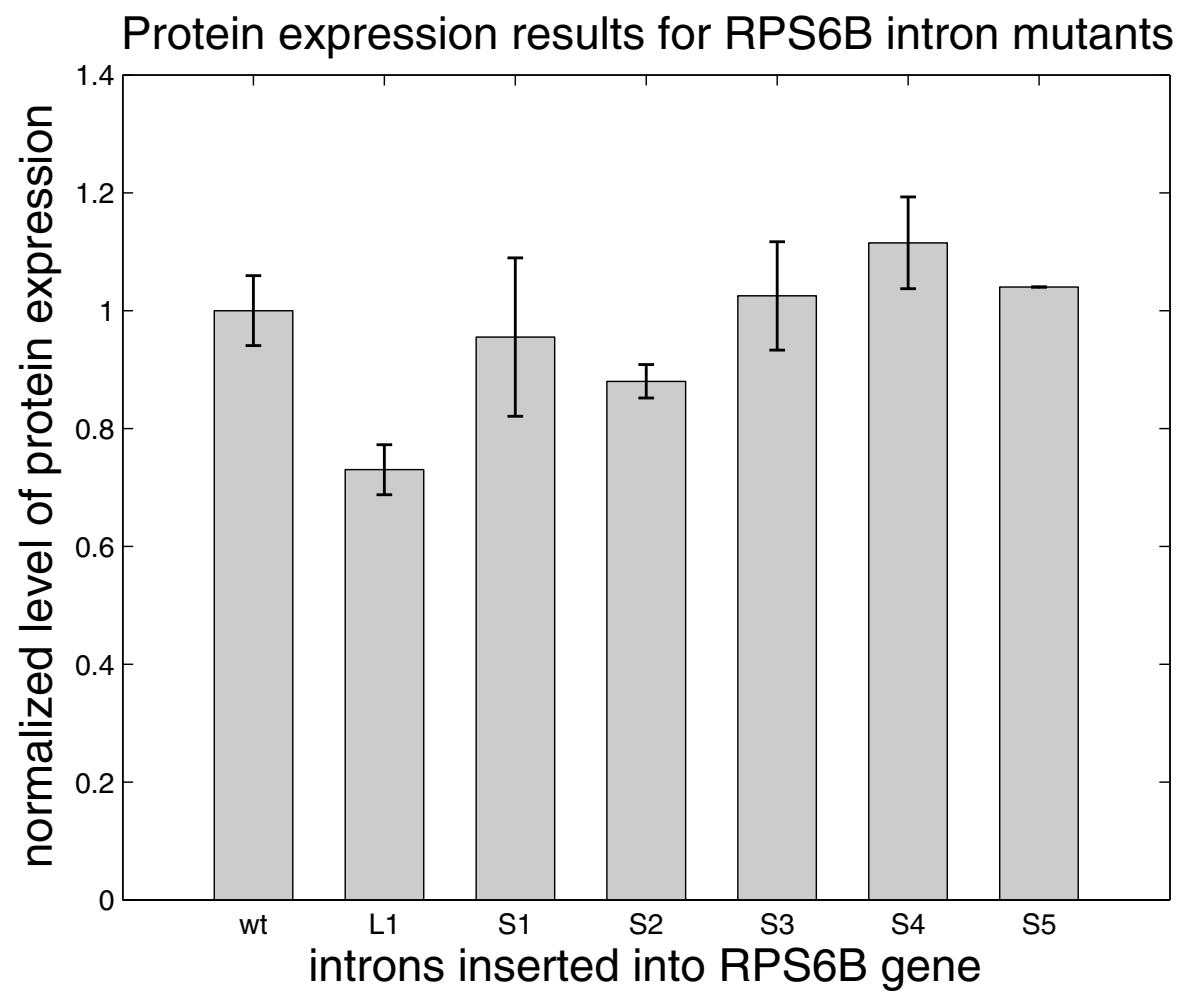

Figure 7

Protein expression results for the RPS6B gene containing the newly designed mutant introns.

since there are no reasonably reliable algorithms for predicting RNA tertiary structure. However, it is believed that RNA secondary structure plays a crucial role in tertiary structure formation, since most tertiary interactions are thought to arise after the formation of a stable secondary structure, when the molecule is able to bend around the flexible, single-stranded regions [34,35]. Moreover, the tertiary structure interactions that arise in the later stages of folding are usually too weak to disrupt secondary structure that has already formed. Therefore, we believe that the structural branchpoint distance based on the second-

Table 7: Characteristics of newly designed APE2 mutants.

\begin{tabular}{ccc}
\hline mutant & avg $\left(\boldsymbol{d}_{s}\right)$ & bp prob \\
\hline wt & 27.90 & 0.37 \\
ape2-LI & 75.73 & 0 \\
ape2-L2 & 69.68 & 0 \\
ape2-SI & 8.93 & 0.82 \\
ape2-S2 & 23.33 & 0.50 \\
ape2-S3 & 24.60 & 0.45 \\
ape2-S4 & 25.14 & 0.42 \\
ape2-S5 & 4.10 & 0.99
\end{tabular}

$\operatorname{avg}\left(\boldsymbol{d}_{s}\right)$ - average structural branchpoint distance; bp prob probability of $\mathbf{d}_{s}<\mathbf{2 0}$. ary structure interactions provides a reasonable approximation of the true spatial distance.

\section{Conclusion}

Our computational study offers further insights into the role of pre-mRNA secondary structure in gene splicing in yeast. We show that it is necessary to consider near-optimal structure predictions to be able to detect structural differences between intron mutants that have different splicing efficiencies. We also propose a novel method for quantifying a distance between two bases in an RNA secondary structure and apply this to compute structural branchpoint distances in the studied intron mutants. Positive experimental results on three different yeast genes suggest that our model of structural requirements for efficient splicing can be applied universally to all 5 'L yeast introns. Additional laboratory experiments are needed to refine the current model by determining the upper bound of the structural branchpoint distance needed for efficient splicing and acceptable thermodynamic stability of the stems adjacent to splicing signals. Considering that several biological studies indicate that shortening of the branchpoint distance, either by formation of secondary structure or by protein interactions, is important for efficient splicing in Drosophila melanogaster and some mam- 


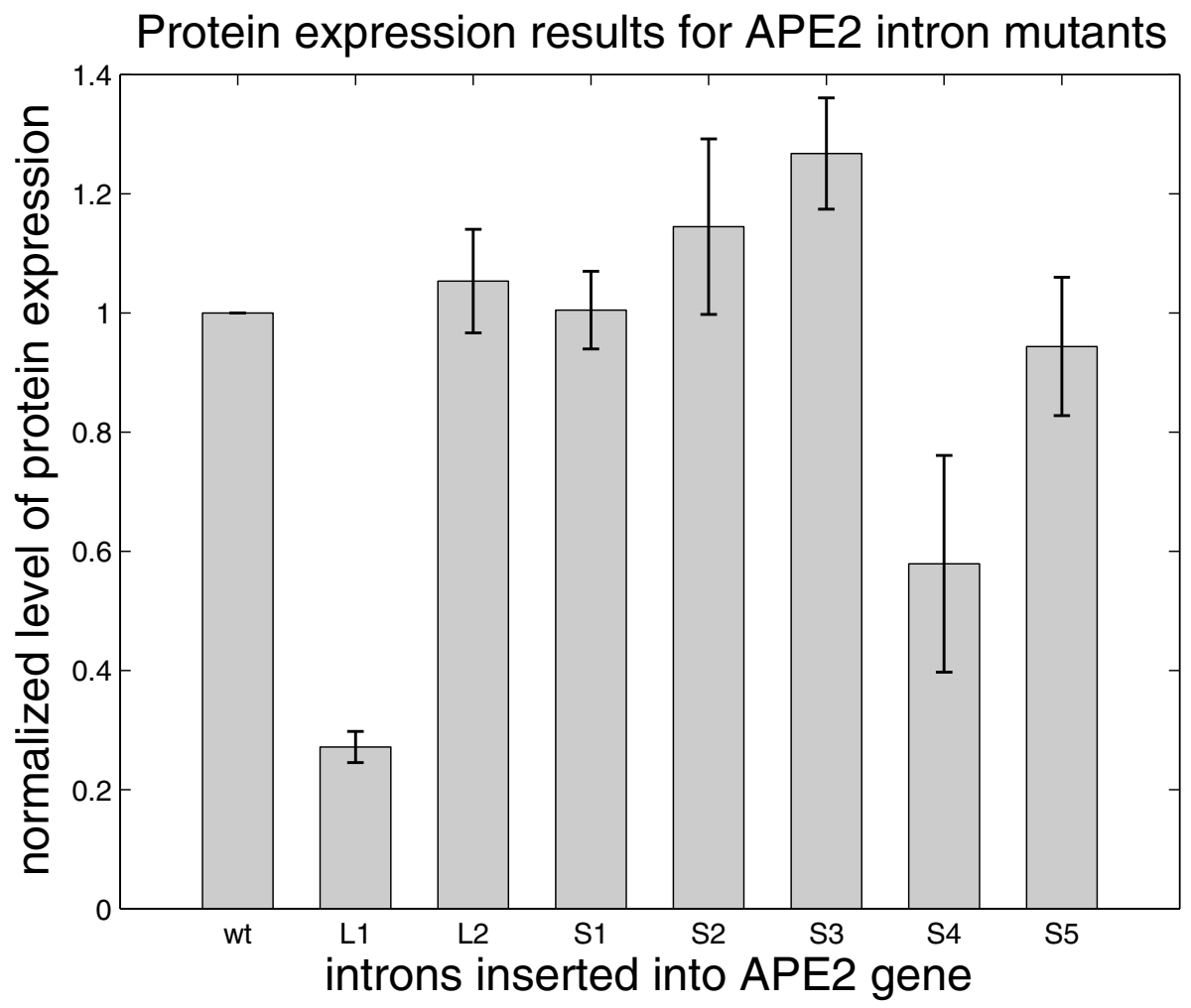

\section{Figure 8}

Protein expression results for the APE2 gene containing the newly designed mutant introns. Protein expression level is normalized with respect to wildtype expression level. Shaded boxes represent the mean value for several different samples and error bars represent +/- I standard deviation for these samples.

malian species [12,13], it might be possible to extend our model to define structural requirements for efficient splicing in other eukaryotes. Another possible application of our findings is in gene-finding, where structural characteristics of identified long introns can be used to distinguish between real and false positive predictions.

\section{Methods}

\section{Computational RNA secondary structure prediction}

In this work we used four different RNA secondary prediction tools: mfold [14,15], RNAsubopt [20], RNAfold [22] and Alifold [26].

Mfold was used for predicting MFE secondary structures for Libri et al.'s [8] mutants and for predicting suboptimal structures within $5 \%$ of the MFE during the mutant design process. Mfold uses dynamic programming to identify the MFE secondary structure and a set of suboptimal structures within a user defined percentage from the MFE for a given RNA sequence. We used both, the web (3.2) and command line (3.0) versions of mfold with default parameters.
RNAsubopt was used to compute a sample of 1000 suboptimal structures within the 5\% from the MFE. Unlike mfold, it computes all suboptimal secondary structures within a user defined energy range or percentage from the MFE for a given RNA sequence. It can also draw a random sample of the computed suboptimal structures using their Boltzmann weights. We used the command line version of RNAsubopt with options "-ep 5 -p 1000 -noLP", which specify the percentage from the MFE (5\%), random sample size (1000) and disable prediction of helices of length 1.

RNAfold was used to compute partition function and base-pair probabilities. RNAfold uses dynamic programming to compute the MFE secondary structure of a given RNA sequence, but when run with option '-p' it also computes base-pair probabilities.

Alifold was used to compute consensus secondary structure for $R P S 17 B$ and RPS $6 B$ introns based on the alignment of introns in Sensu stricto species. It uses modified dynamic programming algorithms that add a covariance 


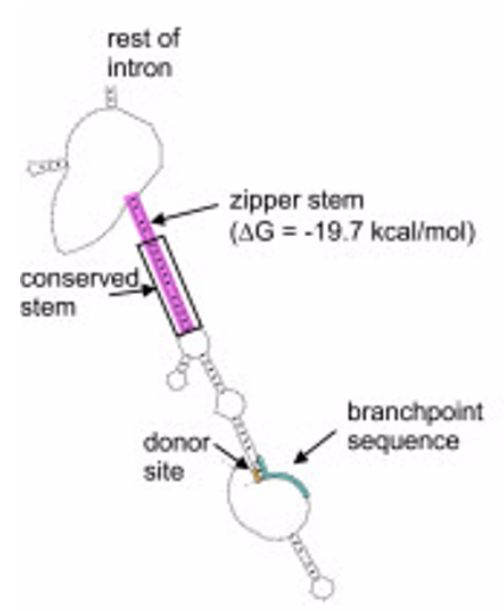

RPS17B

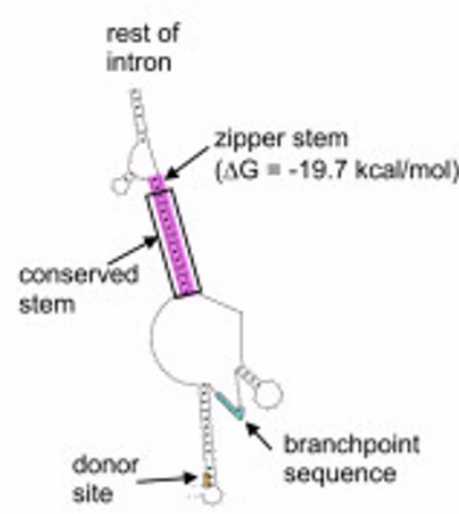

RPS6B

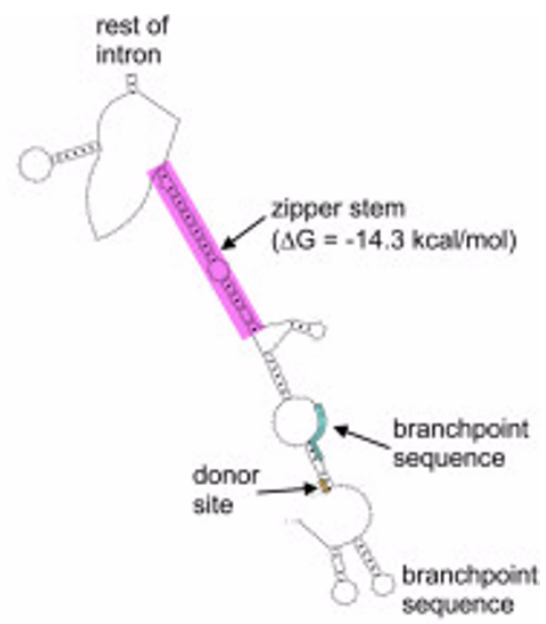

APE2

\section{Figure 9}

Portions of the RPSI 7B, RPS6B and APE2 introns containing computationally identified zipper stems. The free energy values $(\Delta G)$ for the shaded zipper stem are given in parentheses. Stems conserved between Saccharomyces sensu stricto group are also labeled.

term to the standard energy model to compute a consensus secondary structure for a set of aligned RNAs.

RNAsubopt, RNAfold and Alifold are part of the Vienna RNA secondary structure package [22] (we used version 1.7). All four algorithms use free energy calculation based on Turner's nearest neighbour energy model [15,36-38].

\section{Distance calculation in an RNA secondary structure}

Calculating the spatial distance between two nucleotides in a folded RNA molecule requires knowledge of the tertiary structure of the molecule. Since currently there are no reasonably reliable algorithms for predicting RNA tertiary structure, our distance calculation is based solely on RNA secondary structure. Considering that secondary structure is generally believed to play a crucial role in tertiary structure formation [34,35], this approach should give us a good approximation of the true spatial distance.

To calculate the structural branchpoint distance $d_{s^{\prime}}$ we consider a predicted secondary structure of the intronic pre-mRNA as an undirected graph whose vertices are nucleotide bases and whose edges correspond to the bonds between the nucleotides. These bonds can be either sugar-phosphate bonds between the nucleotides in the RNA chain or the hydrogen bonds between paired bases in a given RNA secondary structure. Figure 10 shows the conversion from an RNA secondary structure to the secondary structure graph representing it. To compute the distance between two vertices in the graph, we employed Dijkstra's shortest-path algorithm [39]. Since Dijkstra's algorithm requires a directed graph, we represent each non-directed edge $(u, v)$ as two directed edges, $(u, v)$ and $(v, u)$. All edges in the RNA secondary structure graph have uniform weight $w(u, v)=1$.

In our implementation of the algorithm, the inputs to the program are a pseudoknot-free RNA secondary structure in dot-bracket notation (Vienna format) and the locations of two bases for which the distance needs to be calculated. These bases are the first nucleotide of the intron and the bulging A in the branchpoint sequence (UACUAAC). The output of the program is the shortest distance between these two bases, which we consider as the structural branchpoint distance $\left(d_{s}\right)$ for the given intron secondary structure. The program is available at http://cs.ubc.ca/ $\simeq$ rogic/splicing.html.

\section{Mutant sequences}

We used two basic strategies for designing intron mutants with desired structural characteristics. To obtain mutants with long structural branchpoint distances we aimed to disrupt a zipper stem that was bringing the donor site and the branchpoint sequence close together in the wildtype intron. Conversely, for the mutants designed to have efficient splicing we aimed to stabilize the zipper stem found in the wildtype intron. With these strategies in mind, we used a combination of a trial-and-error approach and secondary structure designs computed by RNA Designer [40] to obtain mutant sequence with desired structural characteristics. 


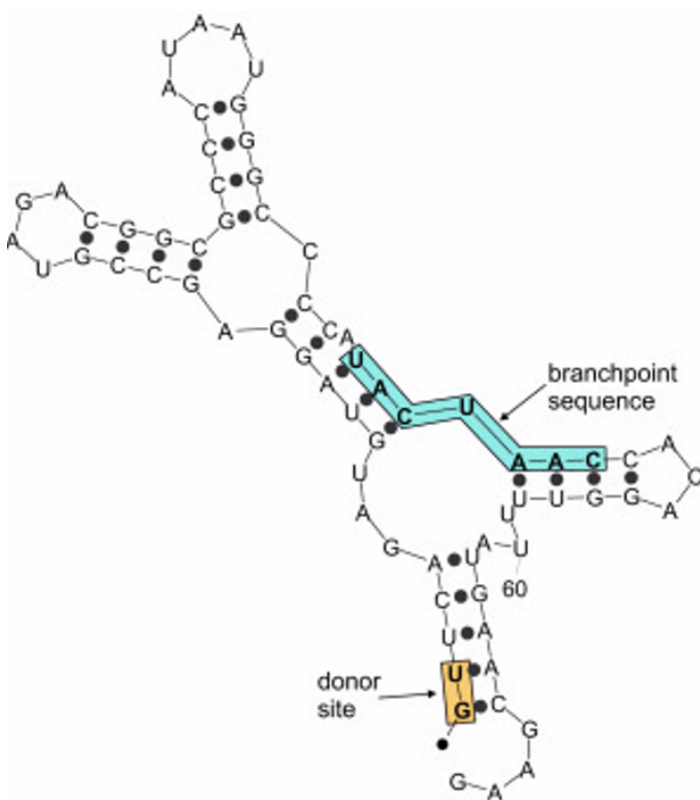

(a)

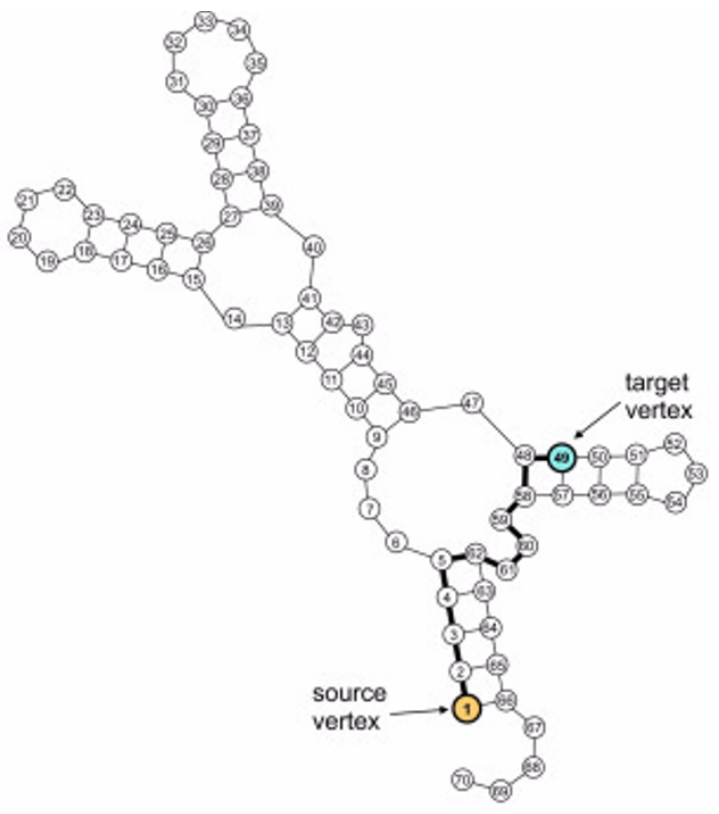

(b)

Figure 10

Conversion from the RNA secondary structure to the graph representing it. (a) Graphical representation of the secondary structure of an intron produced by mfold (filled-in circles represent base-pairing interactions, i.e., hydrogen bonds). (b) Graph representing the RNA structure in (a). The bolded path between the source and target vertices is the one found by the algorithm to be the shortest $\left(d_{s}=I I\right)$.

Most of the intron mutants that we designed have segment substitutions around 20-30 nt long. Sequence segments of this size allowed us to rearrange the secondary structure of a mutant in a desired way. The exception is mutant $r p s 6 b$-S5 which has three short insertions ( $8 \mathrm{nt}$ in total) in the polypyrimidine tract of RPS $6 B$ intron. Mutant $r p s 17 b-L 3$ is a result of two 3-nucleotide-segment substitutions in Libri et al.'s [8] mutant $8 m U B 1$ (the middle sequence of lower case letters represents the original $8 m U B 1$ mutation). Similarly, mutant $r p s 17 b-S 3$ is a result of a 4-nucleotide-segment substitution in the $3 \mathrm{mDB} 1$ mutant (the first segment of lower case letters represents the original $3 m D B 1$ mutation). Table 8 gives the location and sequence of mutant substitutions and Figure 11 depicts mutant locations with respect to the secondary structure of the introns we studied.

\section{Generation and assaying of intron mutants}

Using the TRP1 gene as a selectable marker, RPS17B, $R P S 6 B$ and APE2 were tagged at their genomic locus with a $-13 \mathrm{MYC}$ fragment to generate $\mathrm{C}$-terminal protein fusions in yeast strains derived from a s288c background [41]. Western blotting with a MYC antibody (Covance Research Products) confirmed expression of the correct size protein product in each strain. The intron of the selected gene plus $5^{\prime}$ and $3^{\prime}$ flanking sequences were deleted through homologous recombination with the URA3 selectable marker in each of these tagged strains. Intron DNA containing sequences homologous to regions $5^{\prime}$ and $3^{\prime}$ of the URA3 insertion plus the selected intron mutations were created by PCR. Transformation of these fragments into the appropriate intron deletion strain results in recombination, removal of the URA3 gene, and insertion of the mutant intron sequence. The URA3 gene product leads to cell death when placed on 5-fluoroorotic acid (5-FOA) due to the conversion of 5-FOA to a toxic by product [42]. After transformation, cells can be selected on 5-FOA for those strains that have lost URA3 via insertion of the mutant intron, and thus can grow in the presence of 5-FOA. PCR was used to confirm that 5-FOA resistant strains were the result of insertion of the mutated intron in place of URA3. Each intron mutation was subsequently confirmed by sequencing. Strains containing the correct intron mutations were mated with a strain carrying a 13 MYC epitope tagged protein of a different molecular weight as an internal control and assayed for protein expression levels by western blotting. Western blotting was performed using 20-200 ng of whole cell lysate with a MYC antibody (Covance Research Products) and was quantitated after being developed with ECL Plus Western 
Table 8: Specifications for the new intron mutants used in our study.

\begin{tabular}{|c|c|c|c|}
\hline mutant & segment location & original sequence & substitution/insertion \\
\hline rpsI7b-LI & $258-268(\mathrm{II} \mathrm{nt})$ & UGAAGAGAGGU & augagacaacu \\
\hline rpsI Ib-L2 & 138-157 (20 nt) & GAUUAGAAAACUCCAUUACU & cuuaaguuaguaaauaccuc \\
\hline rps/7b-L3 & $22-47(26 \mathrm{nt})$ & UGAAGCCGGAUAUGAUGGACUGGGC & uuaAGCCGcuacuacuUGGACUGucg \\
\hline rpsI Ib-L4 & $167-189$ (23 nt) & AGAAGAGCGCUCAAUGAAGUAGU & uggcuuggguuaguaggugccuc \\
\hline rpsI7b-SI & $217-23 \mid(15 \mathrm{nt})$ & AAUUGCUUUCGAAUG & uuucauguguucagc \\
\hline rpsI7b-S2 & $280-286(7 \mathrm{nt})$ & UAAGUUG & uacguac \\
\hline rpsI7b-S3 & $246-253(8 \mathrm{nt})$ & AUCCAAUG & uagCggcu \\
\hline rpsI7b-S4 & $244-253(10 \mathrm{nt})$ & UUAUCCAAUG & cuucaucaac \\
\hline rps6b-LI & $21-54(34 \mathrm{nt})$ & CCUUAGAAUUCUAAUGAAUCAGCACGCGCUAACC & guauuuugggugugucccuguuauaaauaauacc \\
\hline rps6b-SI & $19-29$ (II nt) & AUCCUUAGAAU & uuuguuaguaa \\
\hline rps6b-S2 & $87-113(27 \mathrm{nt})$ & CACAAAUUAGUGCACUAUAAUAAAAAU & unauaaauagugauaccauuugguaaa \\
\hline rps6b-S3 & $21-57(37 \mathrm{nt})$ & CCUUAGAAUUCUAAUGAAUCAGCACGCGCUAACCGGC & aaauuccaacguuucccugcaacaugccuuucuuccg \\
\hline rps6b-S4 & $38-55$ (18 nt) & AUCAGCACGCGCUAACCG & auucccaacagacugucc \\
\hline rps6b-S5 & $337-345$ & GUAUUAUUU & GgUguucAUUAUUacaU \\
\hline ape2-LI & $159-175$ (17 nt) & UGUUACCCUCAUAUUCU & ggguacaauuaauagag \\
\hline ape2-L2 & $237-252(16 \mathrm{nt})$ & GCAAUAGCUUAGGUAA & ccuucguacuuuuggg \\
\hline ape2-SI & $23-37(15 \mathrm{nt})$ & CAAAGAAACAAGGAA & agggcagaaauagaa \\
\hline ape2-S2 & $43-57$ (15 nt) & AUACAUAAUAUAAAU & aacugguagguacgu \\
\hline ape2-S3 & $237-252(16 \mathrm{nt})$ & GCAAUAGCUUAGGUAA & caaugaaugagaacuc \\
\hline ape2-S4 & $159-175$ (17 nt) & UGUUACCCUCAUAUUCU & aaauauuaccuaagcua \\
\hline ape2-S5 & $300-322(23 \mathrm{nt})$ & CUCGUUACCGACCUUUGAGUUCU & uuaagcuuuuguguuugagaaca \\
\hline
\end{tabular}

The upper case letters represent the original sequences and the lower case letters represent substitution or insertion sequences. The first base of an intron is numbered $\mathrm{I}$.

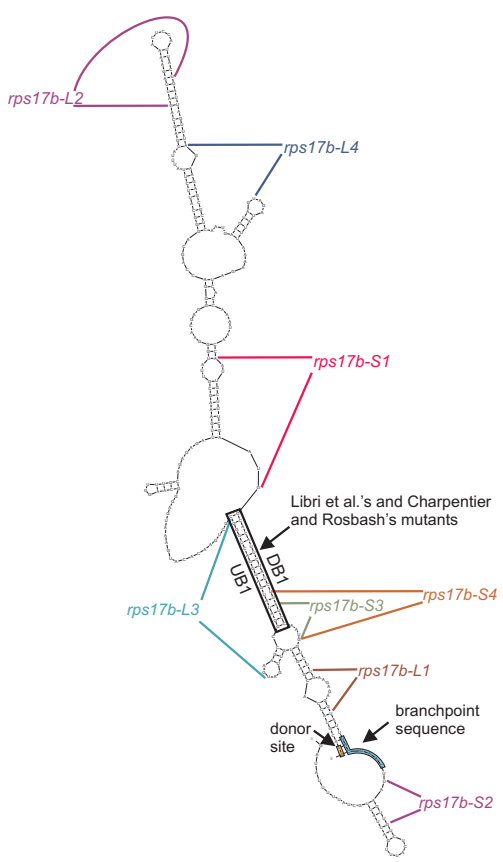

(a)

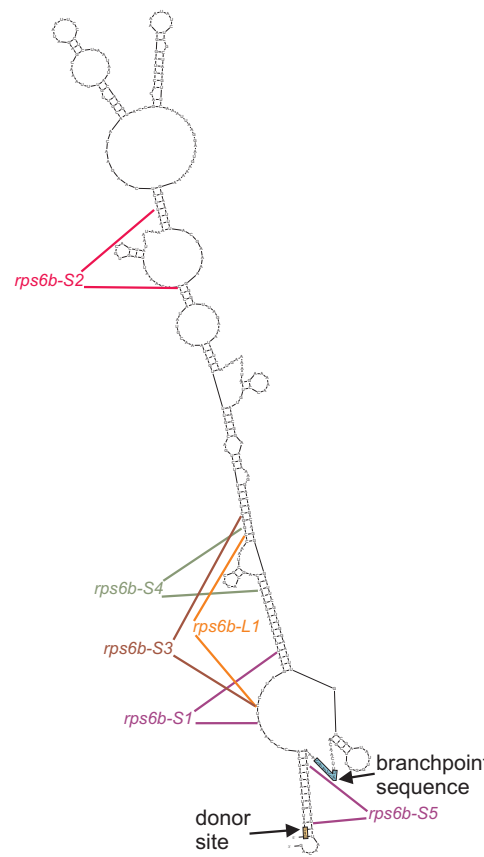

(b)

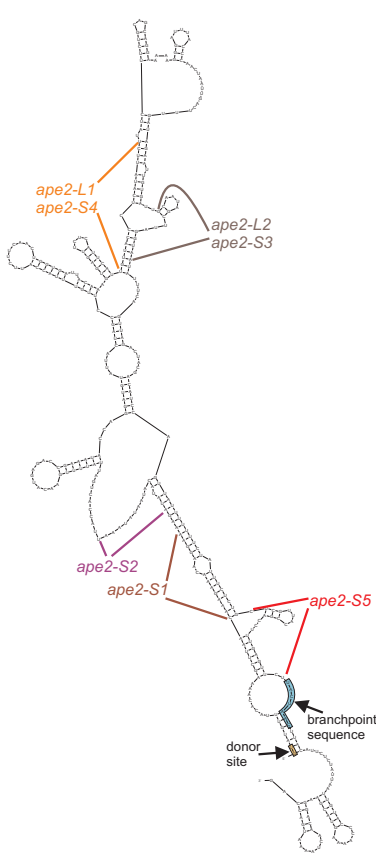

(c)

\section{Figure I I}

Location of mutations with respect to the secondary structure for (a) RPSI 7B, (b) RPS6B, and (c) APE2 introns. The two lines for each mutant indicate the beginning and end of the sequence segment that was modified. 
Blotting Detection reagent (Amersham Bioscience) using a Storm Imaging system (Amersham Bioscience). For each mutant assayed, the internal control was used to normalize protein loading, and the experiments were performed a minimum of 2 times on two independently derived mutant isolates.

\section{Yeast intron dataset}

In order to obtain a high quality yeast intron dataset we consulted three databases: the Ares lab Yeast Intron Database [43], the Yeast Intron DataBase [44], and the Comprehensive Yeast Genome Database [45]. For additional information, we used the Saccharomyces Genome Database (SGD) [46]. We constructed our dataset by including introns that have consistent annotations between at least two of the three databases. We considered only introns from single-intron genes (which represent the majority of intron-containing genes in S. Cerevisiae) that interrupt the gene's coding region (this excluded introns found in the 5' UTR region). The number of introns found to have a consistent annotation between at least two databases was 214 (there are 240 introns in the yeast genome). Eleven of these were excluded because they were not supported by the latest comparative genomic study [23], which labeled them as possible misannotations. The final dataset contains 203 yeast introns, 155 of which are experimentally verified and 48 are putative introns. There are 98 long $\left(5^{\prime} \mathrm{L}\right)$ and 105 short (5'S) introns. We call this dataset the STRuctural INtron (STRIN) dataset. The STRIN dataset is available at http://cs.ubc.ca/ rogic/splicing.html.

\section{Authors' contributions}

SR conceived the study, performed computational experiments and drafted the manuscript. HHH and AKM participated in the design and coordination of the study and together with BFO supervised the research project. BM designed and performed laboratory experiments and helped draft the manuscript. PH helped design laboratory experiments. All authors analyzed the results and reviewed drafts of the manuscript. All authors read and approved the final manuscript.

\section{Additional material}

\section{Additional file 1}

Minimum free energy structures for Libri et al. 's [8] mutants predicted by mfold.

Click here for file

[http://www.biomedcentral.com/content/supplementary/14712164-9-355-S1.pdf]

\section{Additional file 2}

Distribution histograms of structural branchpoint distances for (a) wt, (b) UB1i, (c) DB1i, (d) UB1iDB1i, (e) mut-5, $(f)$ mut-12, and (g) mut18 introns.

Click here for file

[http://www.biomedcentral.com/content/supplementary/1471-

2164-9-355-S2.pdf]

\section{Additional file 3}

Structural characteristics of newly designed RPS17B mutants based on mfold predictions: $\mathbf{d}_{\mathrm{s}}-$ structural branchpoint distances for MFE and all suboptimal predictions within $5 \%$ from the MFE; avg - average $\mathbf{d}_{\mathbf{s}^{\prime}} \boldsymbol{b} \boldsymbol{p}$ prob-base-pairing probability of interaction between the donor site and the branchpoint sequence based on the partition function.

Click here for file

[http://www.biomedcentral.com/content/supplementary/14712164-9-355-S3.pdf]

\section{Additional file 4}

Distribution histograms of structural branchpoint distances for (a) rps17b-L1, (b) rps17b-L2, (c) rps17b-L3, (d) rps17b-L4, (e) rps17bS1, (f) rps17b-S2, (g) rps17b-S3, and (h) rps17b-S4 mutants. Click here for file

[http://www.biomedcentral.com/content/supplementary/14712164-9-355-S4.pdf]

\section{Additional file 5}

Distribution histograms of structural branchpoint distances for (a) RPS6B wildtype intron, (b) rps6b-L1, (c) rps6b-S1, (d) rps6b-S2, (e) rps6bS3, $(f)$ rps6b-S4, and (g) rps6b-S5 mutants.

Click here for file

[http://www.biomedcentral.com/content/supplementary/1471-

2164-9-355-S5.pdf]

\section{Additional file 6}

Distribution histograms of structural branchpoint distances for (a) APE2 wildtype intron, (b) ape2-L1, (c) ape2-L2, (d) ape2-S1, (e) ape2-S2, (f) ape2-S3, (g) ape2-S4, and (h) ape2-S5 mutants.

Click here for file

[http://www.biomedcentral.com/content/supplementary/14712164-9-355-S6.pdf]

\section{Acknowledgements}

We gratefully acknowledge valuable feedback from the four anonymous reviewers, which helped us to improve several aspects of our study.

S. Rogic was supported by an NSERC (the Natural Sciences and Engineering Research Council of Canada) Discovery Grant to A. Mackworth, who also holds a Canada Research Chair in Artificial Intelligence. H. Hoos was partly supported by the Mathematics of Information Technology and Complex Systems (MITACS) Network of Centres of Excellence. B. Montpetit was supported by awards from NSERC and the Michael Smith Foundation for Health Research. P. Hieter is supported by the Canadian Institutes of Health Research (grant MOP-38096) and the U.S. National Institutes of Health (grant POI-CA0I6I5I9).

\section{References}

I. Chow LT, Gelinas RE, Broker TR, Roberts RJ: An amazing sequence arrangement at the 5 ' ends of adenovirus 2 messenger RNA. Cell 1977, 12: I-8. 
2. Berget SM, Moore C, Sharp PA: Spliced segments at the 5' terminus of adenovirus 2 late mRNA. Proc Natl Acad Sci USA 1977, 74(8):3|7|-3I75.

3. Parker R, Patterson B: Architecture of fungal introns: implications for spliceosome assembly. In Molecular biology of RNA: new perspectives Edited by: Inouye M, Dudock BS. San Diego, CA, USA: Academic Press, Inc; 1987: I33-149.

4. Spingola M, Grate L, Haussler D, Ares M: Genome-wide bioinformatic and molecular analysis of introns in Saccharomyces cerevisiae. RNA 1999, 5(2):221-234.

5. Kupfer DM, Drabenstot SD, Buchanan KL, Lai H, Zhu H, Dyer DW, Roe BA, Murphy JW: Introns and splicing elements of five diverse fungi. Eukaryot Cell 2004, 3(5): I088-I I 00.

6. Newman A: Specific accessory sequences in Saccharomyces cerevisiae introns control assembly of pre-mRNAs into spliceosomes. EMBO J 1987, 6( I 2):3833-3839.

7. Goguel V, Rosbash M: Splice site choice and splicing efficiency are positively influenced by pre-mRNA intramolecular base pairing in yeast. Cell 1993, 72(6):893-901.

8. Libri D, Stutz F, McCarthy T, Rosbash M: RNA structural patterns and splicing: molecular basis for an RNA-based enhancer. RNA 1995, I (4):425-436.

9. Charpentier B, Rosbash M: Intramolecular structure in yeast introns aids the early steps of in vitro spliceosome assembly. RNA 1996, 2(6):509-522.

10. Mougin A, Grégoire A, Banroques J, Ségault V, Fournier R, Brulé F, Chevrier-Miller M, Branlant C: Secondary structure of the yeast Saccharomyces cerevisiae pre-U3A snoRNA and its implication for splicing efficiency. RNA 1996, 2(I I): I079-1093.

II. Howe KJ, Ares M: Intron self-complementarity enforces exon inclusion in a yeast pre-mRNA. Proc Natl Acad Sci USA 1997, 94(23): I 2467-I 2472.

12. Chen Y, Stephan W: Compensatory evolution of a precursor messenger RNA secondary structure in the Drosophila melanogaster Adh gene. Proc Natl Acad Sci USA 2003, I 00(20): I | 499-I I504.

13. Martinez-Contreras R, Fisette JF, Nasim FU, Madden R, Cordeau M Chabot B: Intronic binding sites for hnRNP A/B and hnRNP F/ H proteins stimulate pre-mRNA splicing. Plos Biol 2006, 4(2):

14. Zuker M, Mathews DH, Turner DH: Algorithms and thermodynamics for RNA secondary structure prediction: A practical guide. In RNA Biochemistry and Biotechnology NATO ASI Series, Kluwer Academic Publishers; 1999.

15. Mathews DH, Sabina J, Zuker M, Turner DH: Expanded sequence dependence of thermodynamic parameters improves prediction of RNA secondary structure. J Mol Biol 1999, 288(5): $911-940$.

16. Christoffersen RE, Mcswiggen DJ: Application of computational technologies to ribozyme biotechnology products. J Mol Structure 1994, 3 I I:273-284.

17. Betts L, Spremulli LL: Analysis of the role of the Shine-Dalgarno sequence and mRNA secondary structure on the efficiency of translational initiation in the Euglena gracilis chloroplast atpH mRNA. J Biol Chem 1994, 269(42):26456-26463.

18. Freyhult E, Gardner PP, Moulton V: A comparison of RNA folding measures. BMC Bioinformatics 2005, 6:24I-24I.

19. Morgan SR, Higgs PG: Evidence for kinetic effects in the folding of large RNA molecules. J Chem Phys 1996, I 05( I 6):7I 52-7I 57.

20. Wuchty S, Fontana W, Hofacker IL, Schuster P: Complete suboptimal folding of RNA and the stability of secondary structures. Biopolymers 1999, 49(2): |45-165.

21. McCaskill JS: The equilibrium partition function and base pair binding probabilities for RNA secondary structure. Biopolymers 1990, 29(6-7): I I05-III9.

22. Hofacker IL, Fontana W, Stadler LPF, Bonhoeffer S, Tacker M, Schuster $\mathrm{P}$ : Fast folding and comparison of RNA secondary structures. Monatsh Chem 1994, I25:167-188.

23. Kellis M, Patterson N, Endrizzi M, Birren B, Lander E: Sequencing and comparison of yeast species to identify genes and regulatory elements. Nature 2003, 423(6937):24I-254.

24. Saccharomyces sensu stricto alignments [http://wwwgenome.wi.mit.edu/annotation/fungi/comp yeasts/downloads.html]

25. Davis CA, Grate L, Spingola M, Ares M: Test of intron predictions reveals novel splice sites, alternatively spliced mRNAs and new introns in meiotically regulated genes of yeast. Nucleic Acids Res 2000, 28(8): | 700-I 706.
26. Hofacker IL: Vienna RNA secondary structure server. Nucleic Acids Res 2003, 3 I ( I 3):3429-343I.

27. Preker PJ, Guthrie C: Autoregulation of the mRNA export factor Yra Ip requires inefficient splicing of its pre-mRNA. RNA 2006, I 2(6):994-1006.

28. Elliott DJ, Rosbash M: Yeast pre-mRNA is composed of two populations with distinct kinetic properties. Exp Cell Res 1996. 229(2): $181-188$.

29. Kotovic KM, Lockshon D, Boric L, Neugebauer KM: Cotranscriptional recruitment of the $U I$ snRNP to intron-containing genes in yeast. Mol Cell Biol 2003, 23( I 6):5768-5779.

30. Görnemann J, Kotovic KM, Hujer K, Neugebauer KM: Cotranscriptional spliceosome assembly occurs in a stepwise fashion and requires the cap binding complex. Mol Cell 2005, 19:53-63.

31. Zhang Z, Dietrich FS: Mapping of transcription start sites in Saccharomyces cerevisiae using 5' SAGE. Nucleic Acids Res 2005, 33(9):2838-285I.

32. Neugebauer KM: On the importance of being co-transcriptional. J Cell Sci 2002, I I 5(Pt 20):3865-387I.

33. Yu MC, Bachand F, McBride AE, Komili S, Casolari JM, Silver PA Arginine methyltransferase affects interactions and recruitment of mRNA processing and export factors. Genes Dev 2004, I 8( I 6):2024-2035.

34. Brion $P$, Westhof E: Hierarchy and dynamics of RNA folding. Annu Rev Biophys Biomol Struct 1997, 26: I I3-137.

35. Tinoco I, Bustamante C: How RNA folds. I Mol Biol 1999 293(2):27|-28I.

36. Freier SM, Kierzek R, Jaeger JA, Sugimoto N, Caruthers MH, Neil-son $\mathrm{T}$, Turner $\mathrm{DH}$ : Improved free-energy parameters for predictions of RNA duplex stability. Proc Natl Acad Sci USA 1986, 83(24):9373-9377.

37. Turner DH, Sugimoto N, Jaeger JA, Longfellow CE, Freier SM, Kierzek R: Improved parameters for prediction of RNA structure. Cold Spring Harb Symp Quant Biol 1987, 52: I23-I33.

38. Turner DH, Sugimoto N: RNA structure prediction. Annu Rev Biophys Biophys Chem 1988, I7:167-192.

39. Dijkstra EW: A note on two problems in connexion with graphs. Numerische Mathematik 1959, I:269-271.

40. Andronescu M, Fejes AP, Hutter F, Hoos HH, Condon A: A new algorithm for RNA secondary structure design. I $\mathrm{Mol} B \mathrm{Biol}$ 2004, 336(3):607-624

4I. Longtine MS, McKenzie A, Demarini DJ, Shah NG, Wach A, Brachat A, Philippsen P, Pringle JR: Additional modules for versatile and economical PCR-based gene deletion and modification in Saccharomyces cerevisiae. Yeast I998, I4( I 0):953-96 I.

42. Boeke JD, Trueheart J, Natsoulis G, Fink GR: "5-Fluoroorotic acid as a selective agent in yeast molecular genetics". Methods Enzymol 1987, I 54: | 64- I75.

43. Grate $L$, Ares M: Searching yeast intron data at Ares lab Web site. Methods Enzymol 2002, 350:380-392

44. Lopez PJ, Séraphin B: YIDB: the Yeast Intron DataBase. Nucleic Acids Res 2000, 28:85-86.

45. Mewes HW, Frishman D, Güldener U, Mannhaupt G, Mayer K Mokrejs M, Morgenstern B, Münsterkötter M, Rudd S, Weil B: MIPS: a database for genomes and protein sequences. Nucleic Acids Res 2002, 30:31-34

46. Saccharomyces Genome Database [http://www.yeastge nome.org/]

Publish with Biomed Central and every scientist can read your work free of charge

"BioMed Central will be the most significant development for disseminating the results of biomedical research in our lifetime. "

Sir Paul Nurse, Cancer Research UK

Your research papers will be:

- available free of charge to the entire biomedical community

- peer reviewed and published immediately upon acceptance

- cited in PubMed and archived on PubMed Centra

- yours - you keep the copyright 